\title{
Non-equilibrium Phenomena in Superconductors Probed by Femtosecond Time-Domain Spectroscopy
}

\author{
J. Demsar ${ }^{1}$ \\ Received: 4 December 2019 / Accepted: 31 March 2020 / Published online: 29 April 2020 \\ (c) The Author(s) 2020
}

\begin{abstract}
Development of ultrafast lasers and nonlinear optical techniques over the last two decades provides tools to access real-time dynamics of low energy excitations in superconductors. For example, time-resolved THz spectroscopy and time- and angular-resolved photoemission spectroscopy provide access to the real-time dynamics of the superconducting gap amplitude. Such studies enable determination of microscopic parameters like quasi-particle recombination rates, pair-breaking rates and electron-boson coupling constants. Recently, intense $\mathrm{THz}$ pulses have been used to probe the nonlinear dynamics, including observation of collective modes. Moreover, using low-frequency electromagnetic pulses, there are several reports of amplification of superconductivity in both conventional and unconventional superconductors. Starting with a brief historical overview of the pioneering work, where non-equilibrium phenomena in superconductors were investigated using quasi-continuous excitation, we review some of the insights that are provided by using real-time approaches. We focus on conventional BCS superconductors, whose ground state is reasonably well understood, and address similarities and open questions related to the corresponding studies in high- $T_{c}$ superconductors.
\end{abstract}

Keywords Non-equilibrium superconductivity $\cdot$ THz spectroscopy $\cdot$ Electronphonon coupling · Ultrafast phenomena

\section{Introduction}

The field of non-equilibrium superconductivity [1, 2] started soon after the first observation of the superconducting gap using optical spectroscopy [3] and the development of the BCS theory. Given that small energy gap $\Delta$ on the order of a $\mathrm{meV}$ (depending on the material), superconductors were first considered as potential detectors for far-infrared radiation [4]. For such applications, understanding the

\section{J. Demsar}

demsar@uni-mainz.de

1 Institute of Physics, Johannes Gutenberg-University Mainz, Mainz, Germany 
dynamical processes in a superconductor as a function of base temperature, photon energy and excitation density is crucial. On the other hand, even more fascinating observations followed in early 1960s, where superconductivity in Al and Sn was enhanced (increasing the critical current, the superconducting gap and even the critical temperature) under illumination with electromagnetic radiation at sub-gap frequencies [5-8]. Most of the early studies were performed in a quasi-continuous excitation regime, with the characteristic timescales of the processes only estimated. In the last two decades or so, however, the time-resolved techniques, with their ability to probe the evolution of optical constants, electronic band structure, magnetization and crystal structure with femtosecond time resolution contributed to further our understanding of out-of-equilibrium superconductors.

As noted, non-equilibrium superconductivity has been an intriguing field of the modern solid state physics since the 1960s [1]. Here, we first briefly review the results of the fascinating studies performed in 1960s and 1970s-for more details we refer the reader to Refs. $[1,2]$. Then, we turn to studies of real-time dynamics, driven by the rapid development of femtosecond lasers and the related nonlinear optical methods. While the first real-time experiments with picosecond time resolution were performed on conventional superconductor $\mathrm{Pb}$ [9], the field started to blossom following the early studies on cuprate superconductors [10-18]. The first systematic real-time studies of dynamics in conventional superconductors were performed substantially later [19-22]. Such studies still represent a small fraction of the body of work performed on superconductors, still largely dominated by studies of cuprate and pnictide high-temperature superconductors. Nevertheless, we focus on the results obtained on standard s-wave superconductors and address the differences and peculiarities of the results obtained on unconventional high-temperature superconductors after that. For a review, focusing on ultrafast spectroscopy in correlated high-temperature superconductors, we refer the interested reader to a recent review [23].

\subsection{Dynamics Following the Above Gap Excitation of a Superconductor}

We should first note that, in general, there are two types of non-equilibrium that can be realized in superconductors [1], often referred to as the energy-mode and chargemode imbalance. The two types are related to the nature of excitations in superconductors, which continuously changes from a hole-like (far below the Fermi level) to an electron-like (far above the Fermi level) within the energy range of $\approx 2 \Delta$ around the Fermi level.

The first type of nonequilibrium is realized by perturation with charged particles, e.g. by quasiparticle injection through a superconductor-metal junction, resulting in net quasiparticle charge density (note the fractional charge of quasiparticles close to $\Delta$ ). In this case, the populations of the electron-like and the hole-like quasiparticle branches do not coincide, and such a state is referred to as charge imbalance (or branch-imbalance). Correspondingly, there is a difference in the chemical potentials of quasiparticles and the condensate (see Figure 11.1 of Ref. [1]). 
The energy-mode imbalance is realized by exciting a superconductor with charge-neutral drive, like electromagnetic radiation or ultrasound, or with symmetrical tunnel injection in a superconductor-insulator-superconductor junction. Here, the nonequilibrium state is characterized by a (non-equilibrium) distribution function, which is particle-hole symmetric; i.e. the populations of the electron-like and the hole-like branches of the quasiparticle spectrum are the same (see Figure 11.1 of Ref. [1]). Often, this state can be approximated by an effective quasiparticle temperature $T^{*}$. It is the energy-mode imbalance that is considered in the case of photoexcitation discussed in this work.

The first non-equilibrium studies/effects were observed using injection of quasiparticles through tunnel barriers [24], or by (quasi-)continuous laser irradiation [25]. Here, photo-excitation with light, whose frequency is larger than $2 \Delta$, breaks Cooper pairs and gives rise to excess quasiparticle (QP) density.

Experiments in multi-tunnel-junction configuration provided first estimates of lifetimes of excited QPs. While radiative recombination lifetime was theoretically estimated for $\mathrm{Pb}$ to $\approx 0.4 \mathrm{~s}$ at $2 \mathrm{~K}$ [4], tunnel-junction experiments provided the upper limit for the average QP lifetime in $\mathrm{Pb}$ to be of the order of $0.2 \mu \mathrm{s}$ at $1.44 \mathrm{~K}$ [24]. It was argued that the relaxation proceeds via QP recombination, where QP pairs recombine to form Cooper pairs by the emission of phonons with $\hbar \omega>2 \Delta$ $[24,26]$. We note again that these experiments were performed under the constant drive, with the relaxation time estimates based on a series of underlying assumptions [24, 26]. One of the first systematic temperature-dependent studies using QP injection was performed in Al [27]. The authors noted that the errors of the extracted relaxation times are relative only (due to the fact that the extracted absolute values of relaxation time depend on the choice/uncertainty of the model parameters used) [27, 28]. Despite the uncertainty in the absolute values, the results revealed that at temperatures far below $T_{c}$ the recombination rate followed an exponential temperature dependence with $\tau_{R}^{-1} \propto \exp \left(-0.3 \Delta / k_{B} T\right)$ [27]. This behavior (apart from the factor 0.3 in the exponent) could be accounted for by considering that the relaxation of the $\mathrm{QP}$ density, $n_{\mathrm{QP}}$, reflects the bi-particle recombination process (see Fig. 1a).

In this case, the dynamics of excess QP's is given by the bi-molecular rate equation
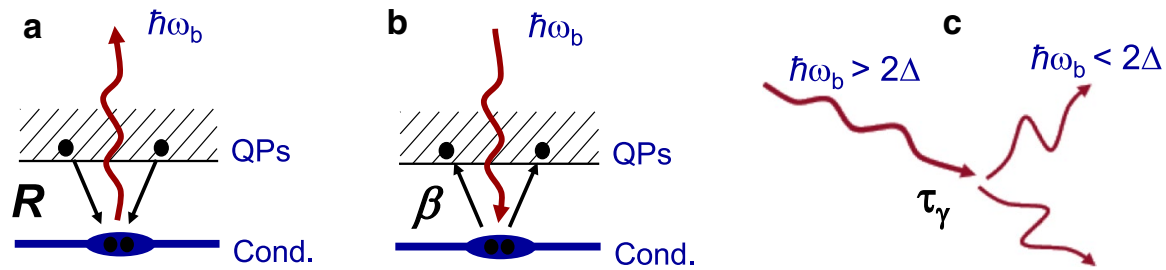

Fig. 1 Different processes governing the relaxation dynamics of photo-excited QPs in a superconductor: a recombination of two QPs into the condensate via emission of $2 \Delta$ boson (phonon), b pair-breaking by absorption of a boson, $\mathbf{c}$ decay of high energy bosons. The latter can proceed via decay of bosons into the (dielectric) substrate of via anharmonic decay into low-energy bosons, with energy less than $2 \Delta$ (Color figure online) 


$$
\frac{\mathrm{d} n_{\mathrm{QP}}}{\mathrm{d} t}=-R n_{\mathrm{QP}}^{2}
$$

where the recombination rate $R$ is a microscopic parameter. These studies were performed in the limit of weak perturbations, where the density of injected (excess) $\mathrm{QPs}, n_{\mathrm{QP}}^{\prime}$, is small compared to the density of thermally excited QPs, $n_{\mathrm{QP}}^{T}$. With $\eta_{\mathrm{QP}}=n_{\mathrm{QP}}^{T}+n_{\mathrm{QP}}^{\prime}$ and $n_{\mathrm{QP}}^{\prime} \ll n_{\mathrm{QP}}^{T}$, the above rate equation can be approximated by $\frac{\mathrm{d} n_{\mathrm{PP}}}{\mathrm{d} t}=-2 R n_{\mathrm{QP}}^{T} n_{\mathrm{QP}}^{\prime}$. It is thus clear that the exponential temperature dependence of relaxation time can be attributed to the temperature dependence of the thermally excited QPs, $n_{\mathrm{QP}}^{T} \propto \sqrt{2 \pi \Delta k_{B} T} \exp \left(-\Delta / k_{B} T\right)$.

We should note that most of these initial studies assumed that the re-absorption of $\hbar \omega>2 \Delta$ phonons (Fig. 1b) emitted during the QP recombination may be neglected. If these high energy phonons rapidly decay into the substrate, or via anharmonic phonon decay (Fig. 1c), it will be the bi-particle recombination process that will be governing the recovery of the SC state. It was Rothwarf and Taylor [29] that pointed out that in general, phonon re-absorption cannot be neglected (including the cases of high excitation densities). The slow decay of the high frequency phonon populations leads in this case to the so-called phonon bottleneck [29].

One of the first real-time studies of suppression of superconductivity with light pulses was performed by Testardi [30]. He studied changes in resistivity in optically thin lead films upon excitation with $\mu s$ pulses. He demonstrated that the absorbed energy density required to suppress superconductivity is substantially lower than the thermal energy, required to heat up the superconductor up to $T_{c}$. To account for the observation, Owen and Scalapino proposed the so-called $\mu^{*}$ model [31], where under continuous excitation the QPs, the condensate and the lattice are in thermal equilibrium, yet the chemical potentials of QPs and the condensate are different. This assumption was motivated by considering the thermalization times being faster than the QP recombination times. According to the $\mu^{*}$ model, upon increasing the excitation density, the superconductor should undergo a first order phase transition into the normal state. Considering the idea that it is the escape of high energy phonons that governs the recovery of the superconducting state [29], and following the experimental work of Sai-Halasz at al., who failed to observe the predicted first-order phase transition [32], Parker suggested an alternative model [33]. He argued that in out-of-equilibrium the QPs and the condensate share the same chemical potential. However, their temperature $T^{*}$, which is the same as the temperature of $\hbar \omega>2 \Delta$ phonons, differs from the temperature of low energy $(\hbar \omega<2 \Delta)$ phonons (base temperature). While it was argued that the above mentioned $T^{*}$ and $\mu^{*}$ models may not adequately describe the behavior in the vicinity of the phase transition [34], the solutions of kinetic equations clearly show non-equilibrium effects in phonon population, consistent with the idea put forward by Parker (see, e.g. Figure 8 of Ref. [34]).

\subsection{Enhancement of Superconductivity with Sub-gap Excitation}

As mentioned above, studies of superconducting properties under continuous irradiation with sub-gap radiation (e.g. microwaves) showed surprising results [5-8]. These 
are illustrated in Fig. 2, summarizing observations of microwave enhancement of superconductivity in superconducting thin-film strips of Al [7]. It was demonstrated that at temperatures close to the equilibrium critical temperature and for frequencies of microwave radiation above some characteristic value, one can observe a measurable increase in the superconducting critical current (Fig. 2a), as well as an increase in the superconducting critical temperature and the magnitude of the superconducting gap. In these experiments, the order parameter jumped discontinuously to zero at the superconducting transition (see Fig. 2b) [7, 35]. With microwave irradiation, superconductivity could be observed above the equilibrium critical temperature, a phenomenon that is commonly referred to as photo-induced superconductivity.

These observations were explained by Eliashberg [36, 37], who realized that in the vicinity of the critical temperature, the effect is mainly related to a non-equilibrium distribution of quasiparticles induced by the microwave field. In particular, at finite temperatures, sub-gap radiation couples to thermally excited QPs at the gap edge, giving rise to excitation of quasiparticles to energies further from the gap edge. Within the BCS theory, the superconducting gap is determined by the self-consistent temperaturedependent gap equation:

$$
\frac{1}{N(0) V_{0}}=\int_{0}^{\hbar \omega_{c}} \mathrm{~d} \xi \frac{\tanh \left(\frac{1}{2} \beta \sqrt{\xi^{2}+|\Delta|^{2}}\right)}{\sqrt{\xi^{2}+|\Delta|^{2}}},
$$
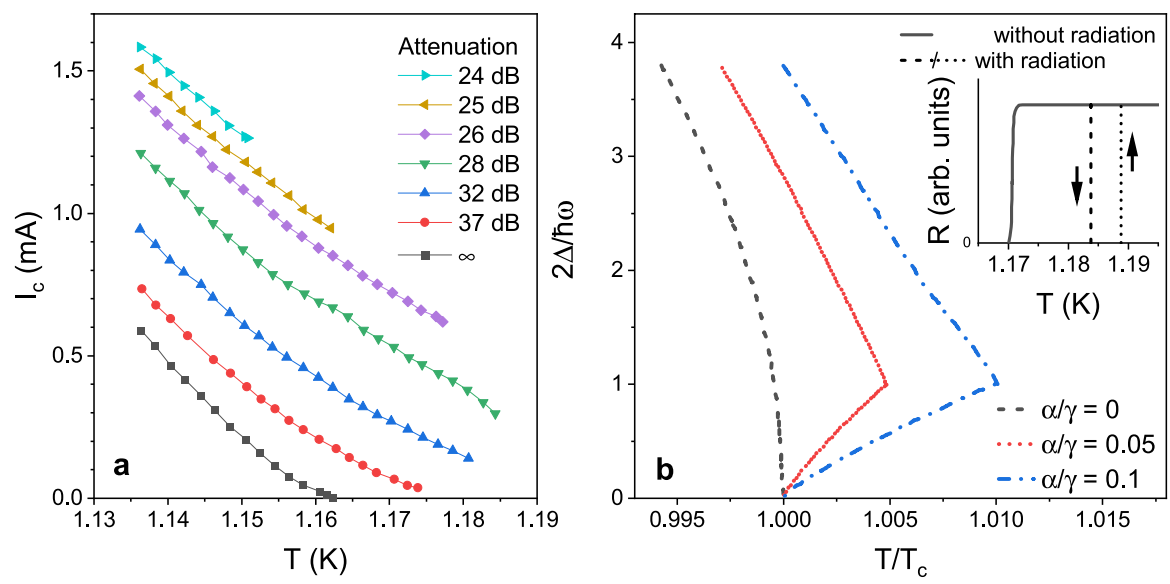

Fig. 2 a Temperature dependence of the critical current in Al for various levels of microwave power at $3 \mathrm{GHz}$ (attenuations are shown on the plot, with $\infty$ corresponding to the absence of microwave radiation). The results show not only a microwave enhanced critical current, but also an increase in the superconducting transition temperature. In equilibrium $T_{c}=1.170 \mathrm{~K}$, while under microwave radiation the sample superconducts already at $1.184 \mathrm{~K}$. b The calculated temperature dependence of the superconducting gap in $\mathrm{Al}$ under $3 \mathrm{GHz}$ radiation using the Eliashberg theory. Inset presents the resistivity curves with and without microwave radiation (for the latter, the hysteresis demonstrates the first order nature of the phase transition under microwave excitation). The presented data were adapted from Ref. [7] (Color figure online) 
where $\xi$ is the kinetic energy relative to the Fermi level. The maximum gap enhancement would result by exciting thermally excited QPs as far as possible from the gap-edge. Using Eq. (2) and expanding for small changes of the order parameter $|\Delta|^{2}=\left|\Delta_{0}\right|^{2}\left(1+\delta \Delta /\left|\Delta_{0}\right|\right)$ as a result of changes in the Fermi-Dirac distribution, with $f(\xi)=f_{\mathrm{FD}}\left(\sqrt{\xi^{2}+|\Delta|^{2}}\right)+\delta f(\xi)$, where $f_{\mathrm{FD}}$ is the Fermi-Dirac distribution and $\delta f(\xi)$ is the excitation induced change in the distribution function, one obtains (in the limit of small relative gap changes) [38]

$$
\frac{\delta \Delta}{\left|\Delta_{0}\right|} \approx-C \int_{0}^{\hbar \omega_{c}} \mathrm{~d} \xi \frac{\delta f(\xi)}{\sqrt{\xi^{2}+\left|\Delta_{0}\right|^{2}}} .
$$

Here, $C$ is a positive dimensionless constant that generally depends on the base temperature. From Eq. (3), it follows that pushing the gap-edge quasiparticles to higher energies, $\delta f(0)<0$, results in $\delta \Delta>0$.

Since these experiments were performed using continuous microwave radiation, while inducing changes in the distribution functions require slow thermalization of QPs, it is no surprise that enhancement was observed only for pump frequencies exceeding the characteristic QP scattering rate. Moreover, the observed enhancements of the gap and critical temperature were quite small (e.g. an increase of $T_{c}$ from 1.170 to $1.184 \mathrm{~K}$ in $\mathrm{Al}$-see Fig. 2).

Detailed experimental studies as a function of frequency, power and base temperature showed some departures from the Eliashberg theoretical predictions [35, 39]. Chang and Scalapino [40-42] proposed an extension of the Eliashberg theory by considering the energy dependence of the QP recombination rate (in Fig. 1a the recombination rate $R$ is considered to be a constant, but in general it depends on QP energy). The high energy quasiparticles recombine into Cooper pairs faster, since there are more available phonon states to relax the energy to. By analyzing the coupled Boltzmann equations for phonon and quasiparticle distributions under sub-gap excitation [42], Chang and Scalapino demonstrated cooling of a superconductor upon photo-excitation.

Finally, not only microwave radiation, but also excitation with sub-gap microwave phonons was demonstrated to give rise to enhancement of superconductivity [43-45], which could be explained within the generalized Eliashberg model [46].

For further reading on experimental and theoretical approaches to realize enhancement of superconductivity using quasi-equilibrium sub-gap excitation, we refer to several reviews to $[47,48]$.

\section{Real-Time Spectroscopy of the Superconducting Gap Dynamics}

To study the dynamics of light-induced suppression and recovery of the superconducting state in real-time the common approach is to use the so-called pump-probe spectroscopy [49]. In the simplest version, femtosecond optical pulses of the same photon energy are used for excitation (pump) and for probing the induced changes in optical constants (probe) as a function of time delay between the two. Most of the early studies were performed in this configuration [10-15, 18, 50-57]. While the 
photo-induced changes at optical (probe) frequencies do reflect, via Kramers-Kronig relations, the photo-induced changes and dynamics of the low-energy excitation spectrum (e.g. the superconducting gap), probing the gap dynamics using far-infrared $(\mathrm{THz})$ pulses $[9,16,17,19-22,58-62]$ or with photoemission [63-69], is much more straightforward to interpret.

In the following, we first focus on phenomena observed on a conventional BCS superconductor $\mathrm{NbN}$ obtained using time-resolved $\mathrm{THz}$ spectroscopy [22]. We note that similar results were obtained in another conventional BCS superconductor $\mathrm{MgB}_{2}$ [20], as well as in an electron-doped cuprate superconductor $\operatorname{Pr}_{2-x} \mathrm{Ce}_{x} \mathrm{CuO}_{4}$ [61]. The latter is especially interesting, given the d-wave symmetry of the order parameter in cuprates, as elaborated in Sect. 2.2.

Before discussing the real-time dynamics of superconductors driven by pulsed laser excitation, we should briefly address the characteristic excitation densities. To do so, let us assume a conventional BCS s-wave superconductor, where most of the absorbed energy of optical pulse is used to break Cooper pairs (as elaborately discussed in the following sections this condition seems to be fulfilled in conventional superconductors, while in the high- $T_{c}$ cuprate superconductors the situation may be more complicated). Under this assumption, the absorbed energy density, $A$, should be lower than the superconducting condensation energy, $E_{\mathrm{c}}$, if we are to discuss the dynamics in the perturbative regime (for $A \approx E_{\mathrm{c}}$ excitation results in melting of superconductivity). Here, $A=\frac{F(1-R)}{l_{\mathrm{opt}}}$, where $F$ is the fluence of the incoming optical pulse, typically in the $\mu \mathrm{J} / \mathrm{cm}^{2}$ range, $R$ is the reflectivity of the sample and $l_{\text {opt }}$ is the optical penetration depth. The condensation energy, $E_{\mathrm{c}}$, on the other hand, is-for a conventional BCS s-wave superconductor-given by $E_{\mathrm{c}}=B_{\mathrm{c}}^{2} / 2 \mu_{0}=N(0) \Delta^{2} / 2$, where $B_{\mathrm{c}}$ is the thermodynamical critical field and $N(0)$ is the normal state density of states. The above condition, however, does not yet imply the dynamics to be linear, i.e. excitation density independent. Since the relaxation processes contain bimolecular terms (see Eq. 1), the recovery dynamics will be linear only for excitation densities, where the density of photo-excited quasiparticles is small compared to the density of thermally excited quasiparticles, given by $n_{T} \simeq N(0) \sqrt{2 \pi \Delta k_{B} T} \exp (-\Delta / T)$

\subsection{Photo-Induced Dynamics of the Superconducting Gap in NbN}

Thin $\mathrm{NbN}$ thin films on $\mathrm{MgO}$ substrates, with $T_{c}$ of $15.4 \mathrm{~K}$ were investigated using near-infrared pump THz-probe spectroscopy [70]. Following linear $\mathrm{THz}$ spectroscopy studies (Fig. 3a), where the temperature dependence of the superconducting gap, $\Delta(T)$, is extracted using the fit to the complex optical conductivity [71], $\sigma(\omega)$, the dynamics of the gap following excitation with a $50 \mathrm{fs}$ near-infrared $(800 \mathrm{~nm})$ pulse can be studied. The time evolution of the SC state as a function of time delay, $t$, after optical excitation, was studied by either directly measuring spectrally resolved $\sigma(\omega, t)$ or by measuring the spectrally integrated response by tracking the time evolution of the induced changes in the transmitted electric field, $\Delta E_{\mathrm{tr}}\left(t^{\prime}, t\right)$, at a fixed $t^{\prime}$ (see Fig. 3c). The latter approach is particularly useful for studying dynamics at low excitations. Given the fact that films are optically 

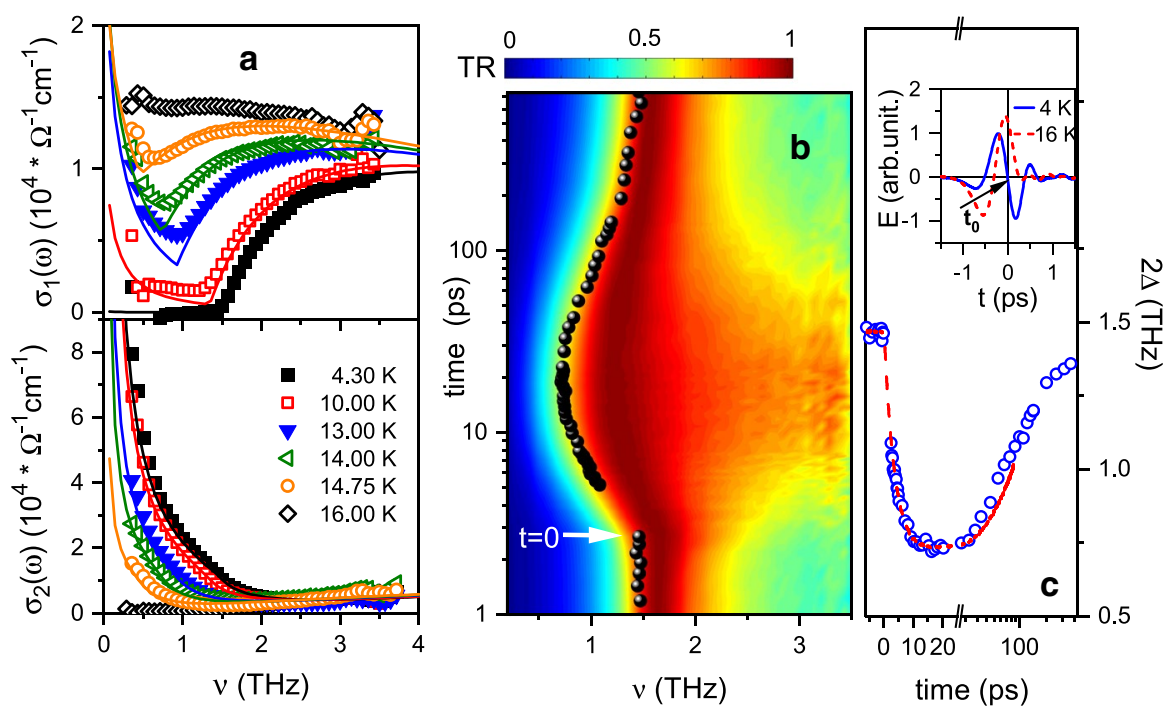

Fig. 3 a The T-dependence of complex optical conductivity, $\sigma(\omega)$, of $15 \mathrm{~nm} \mathrm{NbN}$ film $\left(T_{c}=15.4 \mathrm{~K}\right)$. Solid lines are fits with the BCS equations [71], providing access to the T-dependence of the gap, $\Delta(T)$. b The time $(t)$ evolution of the gap (black spheres), determined at each time delay, $t$, from $\sigma(\omega, t)$. The background contour-plot presents the corresponding ratio of the $\mathrm{THz}$ transmission in the superconducting and normal state, normalized to its peak value. It reflects the time-dependence of the gap. c Presents the gap dynamics, obtained in two configurations: open circles present the values obtained from the analysis of spectrally resolved $\sigma(\omega, t)$, while the dashed line presents the data obtained using spectrally integrated approach. The later, given by the dashed line, is obtained by monitoring the induced changes in the electric field transient at a fixed point of the transmitted $\mathrm{THz}$ electric field transient $\left(t^{\prime}=0\right.$ in the inset) (Color figure online)

thin, they are homogeneously excited. The absorbed energy density, $A$ (in units meV per unit cell volume, meV/ucv), can be determined from the reflectivity and transmission at $800 \mathrm{~nm}$ (pump).

There are several interesting observations in Fig. 3 that should be addressed:

(a) The time-evolution of $\sigma(\omega)$ shows that even at quite high excitation densities as presented here, $\sigma(\omega, t)$ can be well described by the time-evolution of the superconducting gap, $\Delta(t)$. In fact, the data suggest that $\sigma(\omega, t)$ can be approximated by $\sigma\left(\omega, T^{*}(t)\right)$, where at any given time delay (apart from perhaps on the sub-ps timescale) the superconducting state can be approximated by an effective temperature $T^{*}$, similar to the early description by Parker [33].

(b) Despite the fact that the sample is excited by a $50 \mathrm{fs}$ optical pulse, the pairbreaking process (initial decrease of $\Delta$ ) proceeds on a long $10 \mathrm{ps}$ timescale. The observation is surprising, since the $e-e$ thermalization time in metals is assumed to proceed on a sub-ps timescale [72-74]. Thus, one would naively expect the Cooper-pair breaking to be finished within a few $100 \mathrm{fs}$. The observation of slow pair-breaking suggests an alternative scenario, where bosonic excitations (e.g. phonons), generated during the initial decay of hot carriers towards the gap-edge, are responsible for pair-breaking. 
(c) Finally, in $\mathrm{NbN}$ the superconducting state is almost completely recovered on a timescale of a few hundred ps. While the timescale is much shorter than the SC recovery timescales deduced from continuous excitation experiments on $\mathrm{Al}$ or $\mathrm{Sn}[27,28]$, this observation does not prove the early estimates wrong. Based on available data from time-resolved studies on different superconductors, ranging from conventional BCS superconductors to high- $T_{c}$ cuprates, one can conclude that the characteristic SC recovery timescale is roughly inversely proportional to the size of the superconducting gap.

The most interesting and insightful is however following changes in dynamics as a function of base temperature and excitation density. Here, the data reveal that both pairbreaking rate and the superconducting state recovery timescale depend on both, the excitation density and temperature [20, 22, 61, 62]. These dependencies provide access to the nature of the underlying processes and material specific microscopic parameters illustrated in Fig. 1.

From Fig. 3 it follows that in $\mathrm{NbN}[22,62]$ (the same is true for $\mathrm{MgB}_{2}$ [20] and $\left.\mathrm{Pr}_{2-x} \mathrm{Ce}_{x} \mathrm{CuO}_{4}[61]\right)$, the Cooper pair-breaking process $(\approx 10 \mathrm{ps})$ and SC state recovery $(\approx 100 \mathrm{ps})$ are well separated in time, which allows for a detailed view of the underlying microscopic processes based on their temperature and excitation density dependencies. Below we address the two processes in more detail.

\subsubsection{Cooper Pair Breaking Dynamics: Determination of the Electron-Phonon Coupling}

The rise-time dynamics is clearly reflecting the pair-breaking processes (i.e. the initial reduction of condensate density and an increase in the density of QPs). The pairbreaking was found to be temperature dependent (the process is faster as $\mathrm{T}$ is increased) [20], and photo-excitation density dependent (see Fig. 4) [20, 22]. Especially the latter provides strong indications that the delayed pair-breaking is caused by high-frequency phonons, released during the sub-ps relaxation of high energy electrons [74-76].

As shown in Fig. 4, in both $\mathrm{NbN}$ and $\mathrm{MgB}_{2}$, at low excitation densities the pairbreaking can take tens of ps, speeding up upon increasing the excitation density. To account for these observations, in particular the excitation density dependence of the pair-breaking process, we consider the interplay between the condensate/QPs and highfrequency $(\hbar \omega>2 \Delta)$ phonons as proposed by Rothwarf and Taylor [29]. Within the model, the dynamics of the QP and high frequency phonon (HFP) densities, $n$ and $N$, are described by a set of two coupled differential equations [29]:

$$
\mathrm{d} n / \mathrm{d} t=I_{0}+\eta N-R n^{2} \text { and } \mathrm{d} N / \mathrm{d} t=J_{0}-\eta N / 2+R n^{2} / 2-\gamma\left(N-N_{T}\right)
$$

Here $\eta$ is the probability for pair-breaking by HFP absorption, $R$ is the bare QP recombination rate with the creation of a HFP, $N_{T}$ is the concentration of HFP in thermal equilibrium at temperature $T$, and $\gamma$ their decay rate. The rate $\gamma$ could be governed either by anharmonic decay of HFP [15], since $\omega<2 \Delta$ phonons do not have sufficient energy to break Cooper-pairs, or by the diffusion of HFP into the substrate [77]. Finally, $I_{0}$ and $J_{0}$ represent the initial densities of excess QPs and HFPs, which are generated during the avalanche relaxation of hot QPs towards the gap edge. 

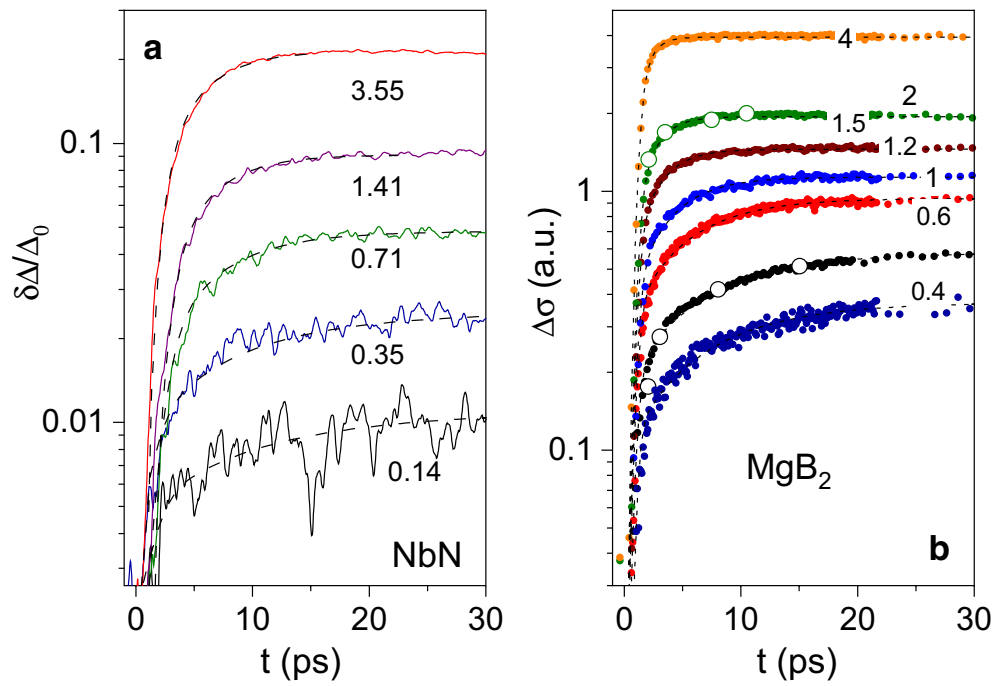

Fig. 4 a Superconducting depletion dynamics shown as the relative change of the gap, $\delta \Delta / \Delta_{0}$, recorded at $4.3 \mathrm{~K}$ on $\mathrm{NbN}$ film for various excitation densities in $\mathrm{mJ} / \mathrm{cm}^{3}$ [22]. The dashed lines are fits to the data with Eq. (7). b Superconducting depletion dynamics $7 \mathrm{~K}$ taken at various fluences (in $\mu \mathrm{J} / \mathrm{cm}^{2}$ ) on $\mathrm{MgB}_{2}$ [20]. Solid circles represent the data obtained by energy integrated approach (see Fig. 3b), while the open circles correspond to $\Delta \sigma(\omega)$ measured directly. Again, the dashed lines are fits to the data using Eq. (7) (Color figure online)

Now, let us imagine that the decay of HFP is the limiting step, governing the recovery of SC, i.e. the so-called phonon-bottleneck scenario by Rothwarf and Taylor [29]. Since the SC recovery dynamics proceed on a much longer timescale (100s of ps), one can-in the first approximation-neglect the decay of HFP given by the last term in Eq. (4), to reduce the equations to

$$
\mathrm{d} n / \mathrm{d} t=I_{0}+\beta N-R n^{2} \text { and } \mathrm{d} N / \mathrm{d} t=J_{0}+\frac{1}{2}\left[R n^{2}-\beta N\right] .
$$

In this case, the early stage of relaxation will result in thermalization of QPs and HFP, which will reach a quasi-equilibrium state, described by a detailed balance equation, $R n_{T^{*}}^{2}=\beta N_{T^{*}}$, where $T^{*}$ will be some new effective temperature (as in the model proposed by Parker [33]). For a SC gap much smaller than the Debye energy and the phonon density of states $D(\omega)=9 v \omega^{2} / \omega_{D}^{3}$ the HFP density is given by $N_{T}=\frac{36 v \Delta^{2} T}{\omega_{D}^{3}} \exp \left(-2 \Delta / k_{B} T\right)$ [15]. On the other hand, the QP density is given by $n_{T} \simeq N(0) \sqrt{2 \pi \Delta k_{B} T} \exp (-\Delta / T)$. This means that at temperatures, where $k_{B} T \ll \Delta$, in equilibrium most of the energy is actually in the QP channel (note the $2 \Delta / k_{B} T$ vs $\Delta / T$ arguments in the exponents). Moreover, from the detailed balance equation it follows that $\eta / R=n_{T}^{2} / N_{T}=\frac{N(0)^{2} \pi \omega_{D}^{3}}{18 v \Delta}$.

It is instructive to investigate the solutions of Eq. (5) for different excitation conditions. For simplicity, we can consider the low temperature limit, where the density of thermally excited QP's and HFP can be neglected. Secondly, we note that the ratio 
of microscopic constants $\eta / R$ has the dimensionality of concentration. Hence, one can introduce dimensionless QP and HFP concentrations, $q \equiv R n / \eta$ and $p \equiv R N / \eta$, while $\theta \equiv \eta t$ is the dimensionless time [78]. With this, Eq. (5) read:

$$
\mathrm{d} q / \mathrm{d} \theta=p-q^{2} \text { and } \mathrm{d} p / \mathrm{d} \theta=-p / 2+q^{2} / 2,
$$

with the initial conditions $p(0)=p_{0}, q(0)=q_{0}$. These coupled equations have analytic solutions $q(\theta)$ and $p(\theta)[20,78]$. Here, we limit the discussion to the dynamics of QP density, $q(\theta)$, which can be directly compared to the experimental data $\Delta(t)$ in Fig. 4. The analytic solution for $q(\theta)$ is

$$
q(\theta)=\left[-\frac{1}{4}-\frac{\xi^{-1}}{2}+\frac{\xi^{-1}}{1-K \exp (-\theta / \xi)}\right],
$$

where $\xi^{-1}=\sqrt{1 / 4+4 p_{0}+2 q_{0}}$ and $K=\frac{\left(4 q_{0}+1\right)-2 \xi^{-1}}{\left.4 q_{0}+1\right)+2 \xi^{-1}}$ are dimensionless constants, depending on the initial conditions $\left(p_{0}, q_{0}\right)$. For long time delays, $\theta \rightarrow \infty$, it follows that $q^{2}(\infty)=p(\infty)$ and $q(\infty)=\frac{1}{4}\left(\sqrt{1+16 p_{0}+8 q_{0}}-1\right)$.

It is further instructive to take a closer look at the meaning of the dimensionless parameter $K$. Obviously, $K=0$ corresponds to the case when immediately after photo-excitation the stationary solution is realized, i.e. when $q_{0}^{2}=p_{0}=q^{2}(\infty)=p(\infty)$. In this case, the time evolution of the QP density, or of the reduction of $\Delta$, would be a step function. The regime $0<K \leq 1$ corresponds to the situation when $q_{0}>q(\infty)$, while $-1 \leq K<0$ represents the $q_{0}<q(\infty)$. For the latter case, $p_{0}>p(\infty)$, which results in an additional pair-breaking until $q^{2}(\infty)=p(\infty)$ is reached.

Figure 5 presents a few simulations of $q(\theta)$ which illustrate different limiting cases. Note that the function (7) was convoluted with the Heaviside step-function with the rise time of $100 \mathrm{fs}$, which accounts for photo-excitation and avalanche initial relaxation/multiplication process of hot quasiparticles. We consider a superconductor with $\Delta=4 \mathrm{meV}$ and the absorbed energy density $A[x \mu \mathrm{eV} / \mathrm{ucv}]$ distributed between QPs and HFPs such that $p_{0}=\frac{R}{\eta} \frac{A(1-x)}{2 \Delta}$ and $q_{0}=\frac{R}{\eta} \frac{A x}{\Delta}$, where $x$ is the fraction of the absorbed energy density in the QP channel $(1 \geq x \geq 0)$.

Figure 5a displays the evolution of the QP density for a constant excitation density and varying $x$, demonstrating the slowly rising signal due to the excess density of HFP (compared to $p(\infty)$ ). It is noteworthy that only for $x \rightarrow 1$ the situation is realized that the QP density is decaying with time in this so-called pre-bottleneck regime. This actually reflects the ratio of $n_{T} \propto e^{-\Delta / k_{B} T}$ and $N_{T} \propto e^{-2 \Delta / k_{B} T}$ discussed above. In other words, for $k_{B} T<<\Delta$ in quasi-equilibrium $n_{T} \gg N_{T}$, and nearly all of the absorbed energy ends up in the QP channel. Therefore it is no surprise that even if only $20 \%$ of the absorbed energy is initially in the HFP channel, the delayed buildup of QP density (or suppression of $\Delta$ ) can be observed.

Panel (b) presents the dependence of pair-breaking on excitation density, with all other parameters being constant. With signal normalized to $A$ the dependence of the apparent pair-breaking time on $A$ reveals the behavior observed in experiments (see Fig. 4). Sub-linearity in the value of $q(\infty)$, i.e. the photo-induced reduction of the gap, at high excitation densities is also revealed. Finally, panel (c) presents the 

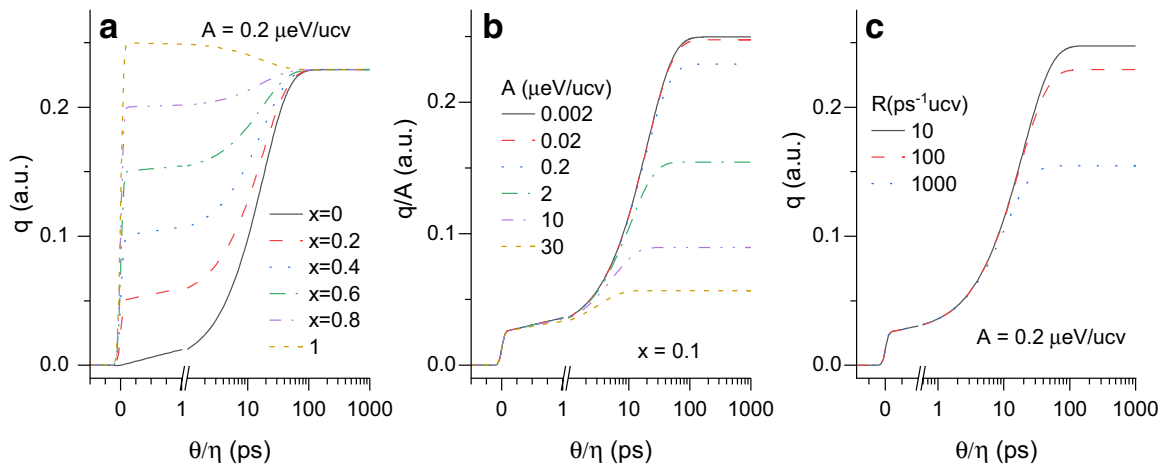

Fig. 5 Simulation of the pair-breaking dynamics using Eq. (7) in the $T \rightarrow 0 \mathrm{~K}$ limit (also valid when photo-excited carrier density much higher than the thermal one). For all simulations $\Delta=4 \mathrm{meV}$ and the pair breaking rate is $\eta=0.1 \mathrm{ps}^{-1}$. a presents the dynamics for constant excitation density, $\mathrm{R}=100 \mathrm{ps}^{-1} \mathrm{ucv}$ and different fractions of the absorbed energy in the QP channel $(0<x<1)$. b Excitation dependence of dynamics for $x=0.1$ and $\mathrm{R}=100 \mathrm{ps}^{-1} \mathrm{ucv}$, note that the signal is normalized to excitation density. $\mathbf{c}$ The dependence of dynamics on the recombination rate and at constant $A$ (Color figure online)

evolution of the dynamics when varying the recombination rate $(R)$ while keeping the pair-breaking rate $\eta$ constant.

Using this analysis on $\mathrm{NbN}$ (the fits to the data are shown by dashed lines in Fig. 4a, where $\Delta$ is known, and it is demonstrated (see Sect. 2.1.2) that the absorbed energy can indeed be simply distributed between the QPs and HFP, one can extract $\eta$ and $K$ and the fraction of the energy density initially stored in the QP channel $x$. The best fit is obtained when $x=0.09$, giving the values of the microscopic constants $\eta^{-1}=6 \pm 1 \mathrm{ps}, R=160 \pm 20 \mathrm{ps}^{-1}$ unit cell.

Since the pair-recombination rate $R$ can be expressed in terms of microscopic parameters through $R=\frac{8 \pi \lambda \Delta^{2}}{\hbar N(0) \Omega_{c}^{2}}[50,79]$, where $\Omega_{c}$ is the phonon cut-off frequency and $N(0)$ is the density of states, the value of $\lambda$ can be determined. Taking the known values for $\Delta, N(0)$ and $\Omega_{c}=16 \mathrm{THz}$ [80], we obtain $\lambda=1.1 \pm 0.12$, which is in excellent agreement with the theoretical estimates, $\lambda=1-1.12$ [81, 82]. Importantly, such an approach does not suffer from the questionable assumptions [74-76] of the so-called two-temperature model, the model commonly used to determine $\lambda$ from time-resolved experiments [72, 73].

\subsubsection{Energetics of the Gap Suppression in Conventional Superconductors}

As addressed above, when at $k_{B} T<<\Delta$ the absorbed energy is distributed between the QPs and HFP and the quasi-equilibrium is reached, most of the energy is in the QP system. This fact provides the means to estimate the condensation energy of a superconductor.

Figure $6 \mathrm{a}$ presents the dependence of the (maximal) relative change of the SC gap $(\delta \Delta / \Delta)$ in $\mathrm{NbN}$ recorded at $4.3 \mathrm{~K}$ as a function of absorbed energy density (in $\mathrm{mJ} / \mathrm{cm}^{3}$ ). At low excitation densities $\delta \Delta / \Delta$ increases linearly with $A$, followed by 
a saturation (plateau at $\delta \Delta / \Delta=1$ is reached when superconductivity is completely suppressed). Following the discussion of the coupled QP and HFP system above, it becomes clear that for the complete collapse of the gap, where QPs would be in a quasi-thermal equilibrium with HFP, the required energy would equal the thermal energy to heat up the sample from the base temperature to above $T_{c}$. This follows from the fact that - in the absence of the gap —all phonons are the HFP (the energy stored in the HFP system in this case will be dominant). On the other hand, the rate at which the gap (or condensate density) gets suppressed in the limit of weak perturbations should provide an estimate of the condensation energy. Alternatively, this may be particularly relevant for bulk samples where the absorbed energy density varies with the depth, an estimate of the characteristic $A$ required to suppress SC, one commonly uses a phenomenological saturation model, $\delta \Delta / \Delta_{0}=\left(1-\exp \left(-A / A_{\mathrm{dep}}\right)\right)$. Here, $A_{\text {dep }}$ is the characteristic absorbed energy density required to deplete SC. It follows from Fig. 6 a that for $\mathrm{NbN} A_{\mathrm{dep}}(4.3 \mathrm{~K}) \approx 25 \mathrm{~mJ} / \mathrm{cm}^{3}$. Within the experimental accuracy, $A_{\text {dep }}(4.3 \mathrm{~K}) \approx 25 \mathrm{~mJ} / \mathrm{cm}^{3}$ is comparable to the thermodynamic SC condensation energy, $E_{\mathrm{c}}$, given by $E_{\mathrm{c}}=B_{\mathrm{c}}^{2} / 2 \mu_{0}=N(0) \Delta^{2} / 2$. Here, $B_{\mathrm{c}}$ is the thermodynamic critical field and $N(0)$ is the electronic density of states at the Fermi level. For $\mathrm{NbN} B_{\mathrm{c}}=0.234 \mathrm{~T}$ [80], giving $E_{\mathrm{c}}=22 \mathrm{~mJ} / \mathrm{cm}^{3}$, nearly identical to $A_{\text {dep }}(4.3 \mathrm{~K})$. Moreover, as shown in Fig. 6b, the T-dependence of $A_{\text {dep }}$ is found to follow the T-dependence of $\Delta^{2}$ (dashed line). We note that the observation in $\mathrm{NbN}$ is in strong contrast to similar studies in cuprates [52,60-61], where $A_{\text {dep }}$ is found to be about one order of magnitude higher than $E_{\mathrm{c}}$ and is nearly independent on temperature [60, $61]$.

\subsubsection{Superconducting State Recovery Dynamics at Temperatures Far Below $T_{c}$}

Now, let us turn our attention to the dependence of the SC recovery time on temperature and excitation density, which is for $\mathrm{NbN}$ shown in Fig. 7.
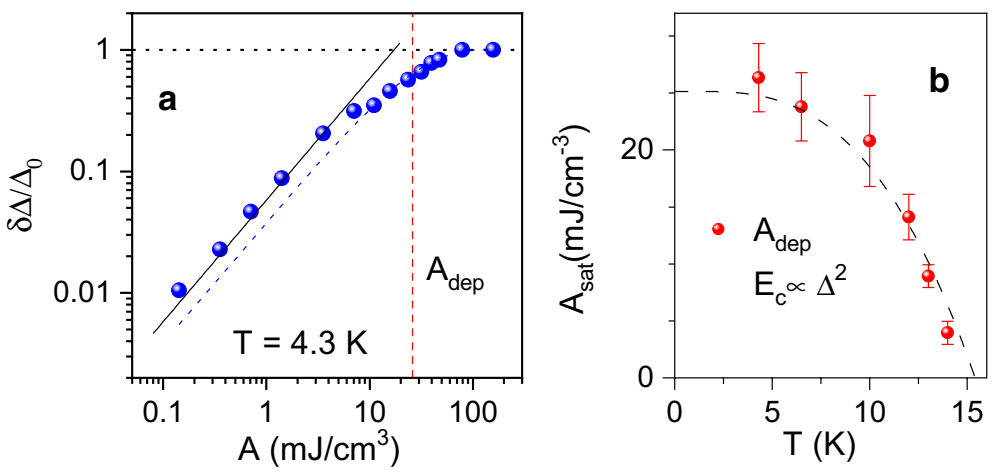

Fig. 6 a The dependence of the peak gap suppression, $\delta \Delta / \Delta_{0}$, in $\mathrm{NbN}$ on $A$ at $4.3 \mathrm{~K}$ (see the traces in Fig. 4a). The dashed line is a fit to the simple saturation model; the solid line is the linear fit. b The T-dependence of the energy density required to deplete superconductivity, $A_{\text {dep }}$, compared to the T-dependence of $\Delta^{2}$ (dashed black line) (Color figure online) 
Figure $7 \mathrm{a}$ presents the relaxation dynamics recorded at $4 \mathrm{~K}$ over 2 orders of magnitude in excitation density. While at the lowest excitation densities the recovery time, $\tau_{\text {rec }}$, substantially exceeds the data acquisition window, one can clearly see speeding up of relaxation dynamic upon increasing excitation density. As shown in Fig. $7 \mathrm{~b}$ the dynamics is below $\approx 10 \mathrm{~K}$ both temperature and excitation density dependent.

As shown in Fig. 7c, at $4 \mathrm{~K} \tau_{\text {rec }}^{-1}$ first increases linearly with $A$, mimicking the intrinsic bi-molecular kinetics of the QP recombination discussed in Sect. 1.1. However, given the fact that the early time dynamics in $\mathrm{NbN}$ reveals a delayed pair-breaking with build-up of quasi-equilibrium between QPs and HFP (see Sect. 2.1.1), it is clear that the recovery dynamics is determined by the decay of the HFP population. That is, the SC recovery dynamics should be described within the phonon bottleneck scenario of Rothwarf and Taylor [29, 78].

Before addressing this, we should note that such excitation density and temperature dependence of SC state recovery was first observed in cuprate superconductors [58, 83, 84], where it was argued to be an evidence for the absence of phonon (boson) bottleneck. It was stated that in the case of a phonon bottleneck the relaxation dynamics should be, at low temperatures, roughly constant and excitation density independent [83, 85]. Surprisingly, and perhaps counterintuitive, considering the full solution of the coupled Rothwarf-Taylor equations, Eq. (4), it has been demonstrated that even in the case of phonon-bottleneck the relaxation should display excitation density and temperature dependence [78] as observed in $\mathrm{NbN}$ (Fig. 7).

While for the complete derivation of analytic solutions for SC state recovery we refer to Ref. [78], we briefly sketch the main arguments here. We remain with the description using dimensionless quantities as above. First of all, including the phonon decay term, the Rothwarf-Taylor equations read
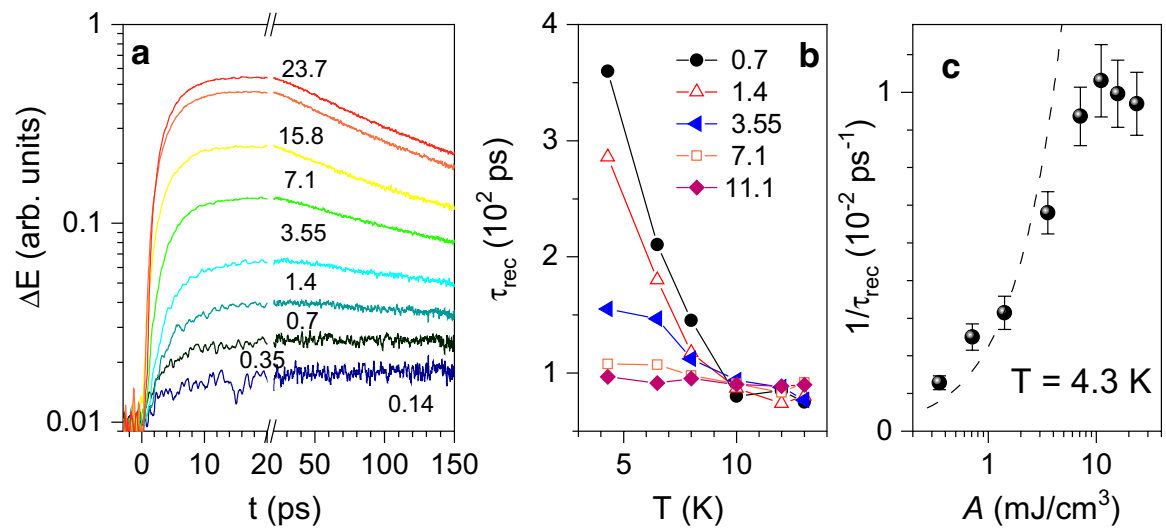

Fig. 7 a The time evolution of the photoinduced gap suppression (spectrally integrated response-see text) recorded on $\mathrm{NbN}$ thin film at $4.3 \mathrm{~K}$ with excitation density covering over two orders of magnitude. b Temperature and excitation dependence of the superconducting state recovery time, $\tau_{\text {rec }}$, obtained by a fit of the data in a with an exponential decay function. c The low temperature superconducting state recovery rate in $\mathrm{NbN}$ as a function of excitation density (Color figure online) 


$$
\mathrm{d} q / \mathrm{d} \theta=p-q^{2} \text { and } \mathrm{d} p / \mathrm{d} \theta=-p / 2+q^{2} / 2-\tilde{\gamma}\left(p-p_{T}\right),
$$

where $\tilde{\gamma} \equiv \gamma / \eta$ is the dimensionless HFP decay rate and $p_{T}$ is the thermal density of HFP. At large time delays, $\theta \geq 1 / \tilde{\gamma}$, both $p(\theta)$ and $q(\theta)$ are obviously slowly decaying, with the decay being determined by the HFP decay. Thus, at large times the difference $q^{2}-p \approx \tilde{\gamma}\left(p-p_{T}\right)$. Considering that the difference $q(\theta)^{2}-p(\theta) \ll p(\theta), q(\theta)$ and that it is decaying slowly, an analytic approximate solution was derived [78], describing the recovery dynamics:

$$
-2 \tilde{\gamma} \theta=\left(2+\frac{1+2 \tilde{\gamma}}{2 q_{T}}\right) \ln \left[\frac{q-q_{T}}{q(\infty)-q_{T}}\right]+\left(2-\frac{1+2 \tilde{\gamma}}{2 q_{T}}\right) \ln \left[\frac{q+q_{T}}{q(\infty)+q_{T}}\right]
$$

Here $q_{T}$ corresponds to the thermal QP density at base temperature and $q(\infty)$ is the value after the initial pre-bottleneck dynamics. Combining solutions for pre-bottleneck case and recovery dynamics an approximate solution for $q(\theta)$, valid over the entire time delay has been derived [78], where

$$
q(\theta)=q_{1}(\theta)+q_{2}(\theta)-q(\infty),
$$

with $q_{1}(\theta)$ given by Eq. (7) and $q_{2}(\theta)$ by Eq. (9). The comparison of the analytic solution, given by Eq. (10), to the numerical solution of Eq. (8), shown in Fig. 8, manifests an excellent agreement for all different limiting cases.

Figure 8 shows that, irrespective of the regime (strong or weak bottleneck), the SC state recovery dynamics displays excitation density dependence at low temperatures. Indeed, at $T \rightarrow 0 \mathrm{~K}$, the time dependence of the inverse of the photo-induced QP density is a linear function of $\theta$, mimicking the pure bimolecular kinetics. At higher temperatures, the relaxation becomes nearly exponential (dashed curve in Fig. 8d presents the exponential decay fit). We note that similar behavior was reported in cuprates [58].
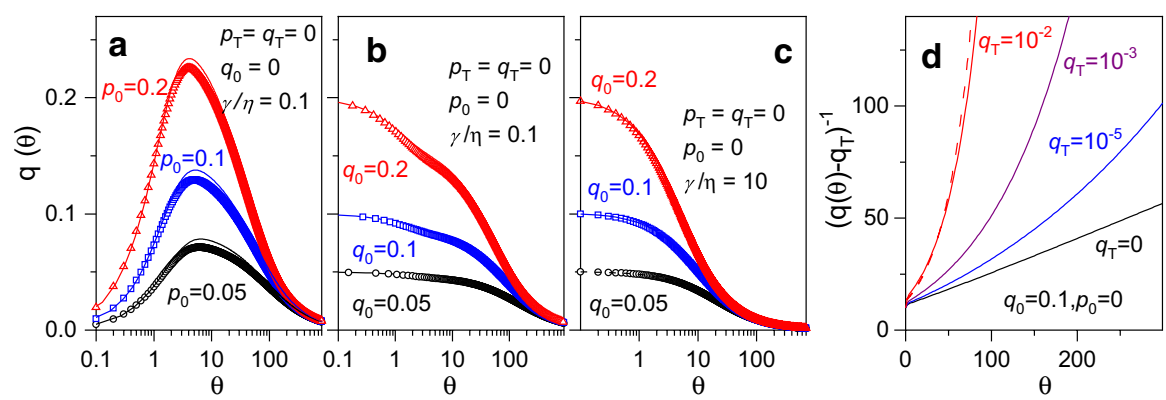

Fig. 8 Analytic solutions of Eq. (10) (open symbols) compared to numerical solutions (solid lines) in several limiting cases. a, b Represent the strong bottleneck limit $(\tilde{\gamma} \equiv \gamma / \eta=0.1)$ for the two extreme excitation conditions ( $x=0$ and 1, respectively) at $T=0 \mathrm{~K}$. c Presents the case of weak bottleneck, i.e. when $\gamma / \eta=10$, at $T=0 \mathrm{~K}$. In all cases, the relaxation dynamics shows a strong dependence of the SC recovery rate on excitation density. Finally, $\mathbf{d}$ presents the dependence of the recovery dynamics on temperature, expressed by the inverse of the photo-induced QP density as a function of $\theta$ for various $q_{T}$. Reproduced from Ref. [78] (Color figure online) 
For the case of a strong bottleneck $(\gamma / \eta \equiv \tilde{\gamma} \ll 1)$, the initial relaxation rate $\tau_{\text {rec }}^{-1}$ is given by [78] (the expression here is valid for $k_{B} T \ll \Delta$ )

$$
\tau_{\mathrm{rec}}^{-1}=\frac{2 \tilde{\gamma}\left(q(\infty)+q_{T}\right)}{(1+2 \tilde{\gamma})}
$$

It is clear that this expression qualitatively accounts for the observations in $\mathrm{NbN}$ (Fig. 7). In the limit of very weak excitations, where $q(\infty) \approx q_{T}$, the relaxation time should display exponential temperature dependence with $\tau_{\text {rec }}^{-1} \propto q_{T}$. Indeed, this accounts also for the early studies of temperature dependence of the relaxation time using biased tunnel junctions [27, 28].

Moreover, the intensity dependence is observed if $q(\infty) \gg q_{T}$. In this case $\tau_{\text {rec }}^{-1}$ is proportional to $q(\infty)$, which is in turn proportional to the excitation intensity (see Fig. 5). It follows that the excitation density dependence of the relaxation time should be observed at low-temperatures, as demonstrated in cuprates [58, 61,83 ] as well as in conventional SCs like $\mathrm{NbN}$ [22], and $\mathrm{MgB}_{2}$ [86].

Further argument for the validity of the Rothwarf-Taylor description of the light-induced gap dynamics in superconductors follows from the comparison of the experimental results on $\mathrm{NbN}$ (Fig. 7) with the model predictions. The data on $\mathrm{NbN}$ show that the excitation and temperature dependence of $\tau_{\text {rec }}$ is observed for $A \leq 5 \mathrm{~mJ} / \mathrm{cm}^{3}$ and for $T \leq 10 \mathrm{~K}$. For higher $A$ and $T \geq 10 \mathrm{~K} \tau_{\text {rec }}^{-1}$ is constant at $\tau_{\text {rec }}^{-1}(0) \approx 0.01 \mathrm{ps}^{-1}$, likely governed by the escape of the HFP into the substrate (since it was found to be inversely proportional to the film thickness [87]). In a phonon bottleneck case $\tau_{\text {rec }}^{-1}$ is proportional to the density of photo-excited QPs, $n_{\mathrm{PI}}$, if $n_{\mathrm{PI}}$ falls into the range $n_{T}<n_{\mathrm{PI}} \leq \beta / R$. Here, $\beta / R$ is the material-dependent characteristic QP density, below which the Rothwarf-Taylor perturbative description is applicable [78]. Indeed, $A=5 \mathrm{~mJ} / \mathrm{cm}^{3}$ corresponds to $n_{\mathrm{PI}} \approx 0.001$ unit cell ${ }^{-1}$, identical to $\beta / R \approx 0.001$ unit cell $^{-1}$ determined from the analysis of the pair-breaking dynamics [22]. Similarly, the fact that $\tau_{\text {rec }}^{-1}$ is constant above $\approx 10 \mathrm{~K}$ follows from the fact that $n_{T}(10 K) \approx 0.0003 \mathrm{ucv}^{-1}$ is comparable to $\beta / R$. Therefore, as experimentally demonstrated for a standard BCS superconductor, the $A$-dependent SC state recovery at low temperatures seems to be an expected behavior for a superconductor driven out of equilibrium.

We note that, while the phenomenological Rothwarf-Taylor has been derived to describe the low-temperature dynamics in superconductors [29, 78], it was demonstrated to be able to qualitatively describe also the dynamics in other systems with small energy gaps near the Fermi level, i.e. when the gap is compared to the cut-off frequency of the coupled bosonic excitation [88, 89]. For example, in heavy fermion metals a dramatic (exponential) slowing down of carrier relaxation was observed at low temperatures [88, 90], originally attributed to slowing down of electron-phonon thermalization [90, 91]. Systematic studies on both heavy fermion metals and Kondo insulators revealed that the dynamics can be accounted for by the presence of the hybridization gap near the Fermi level, with the dynamics of QPs and coupled HFP following the Rothwarf-Taylor bottleneck scenario [89, 91-94]. 


\subsubsection{Superconducting State Recovery Dynamics in the Limit $T \rightarrow T_{c}$}

In the early subsections, we were focusing on the dynamics at low temperatures, i.e. for $k_{B} T \ll \Delta$. Now we address the behavior near $T_{c}$ in the limit when $\Delta \rightarrow 0$. As shown in Fig. 9a, already early experiments using real-time response of the current-voltage characteristic of a tunnel junction to fast laser pulses [96] revealed the anomalous slowing down of relaxation upon approaching $T_{c}$. This can be interpreted by considering the relaxation in terms of the Ginzburg-Landau theory of the secondorder phase transition. Here, the restoring force is given by the derivative of the free energy with respect to the order parameter, giving rise to a divergence of $\tau_{\text {rec }} \propto 1 / \Delta$. Considering the phonons being in thermal equilibrium, Schmid and Schoen derived the expression to describe the dependence of the superconducting state recovery time near $T_{c}$ [97]:

$$
\tau_{\text {rec }}=3.7 \tau_{\mathrm{E}} \frac{k_{B} T}{\Delta},
$$

where $\tau_{\mathrm{E}}$ is the inelastic electron scattering time.

Similarly to early measurements using tunnel junctions, the divergence of superconducting state recovery time was observed in superconductors using optical pump-probe spectroscopy. Typical results obtained on $\mathrm{YBa}_{2} \mathrm{Cu}_{3} \mathrm{O}_{7}$ are shown in Fig. 9b. Assuming the SC state recovery being governed by the decay of HFPs, an alternative scenario, base on the quasi-equilibrium between QPs and HFP was put forward [15]. Here, Kabanov et al. [15] argued that the relaxation rate of the photoinduced QPs is dominated by the energy transfer from HFP $(\hbar \omega>2 \Delta)$ to phonons
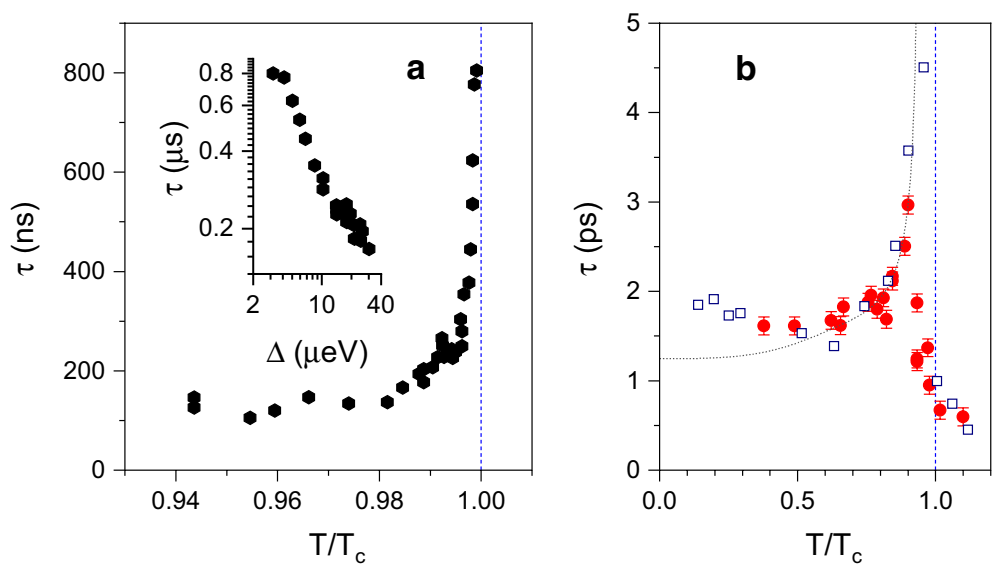

Fig. 9 Dependence of the superconducting recovery timescale in the vicinity of critical temperature. a Relaxation time in superconducting $\mathrm{Al}$ as a function the reduced temperature [96]. The measurement were performed using optically excited tunnel junctions. Near $T_{c}$ the relaxation time shows $1 / \Delta$ dependence (inset). b Relaxation time divergence near $T_{c}$ in photo-excited YBCO high-temperature superconductor. The open squares are from [10], the solid circles are from [95]. The dashed line is a fit using a phonon bottleneck model, i.e. Eq. (13) [15] (Color figure online) 
with $\hbar \omega<2 \Delta$, i.e. by an anharmonic decay of HFP. Considering the kinetic equation for phonons, taking into account phonon-phonon scattering (anharmonicity), the expression describing the relaxation of the equilibrated QP-HFP temperature was derived:

$$
\frac{1}{\tau_{\text {rec }}}=\frac{12 \Gamma_{\omega} \Delta(T)^{2}}{\hbar \omega^{2} \ln \left\{1 /\left(A / 2 N(0) \Delta(0)^{2}+\exp \left(-\Delta(T) / k_{B} T\right)\right)\right\}} .
$$

Here $\Gamma_{\omega}$ is anharmonic phonon decay rate given, e.g. by the phonon linewidth in Raman experiments, $N(0)$ is the normal state electronic density of states, and $A$ the absorbed energy density. Indeed, for $\mathrm{YBa}_{2} \mathrm{Cu}_{3} \mathrm{O}_{7}$ a good agreement of this formula with data (Fig. 9b) is obtained.

Finally, we should mention another aspect of Kabanov's theory [15] , addressing the temperature dependence of the photo-induced QP density in the vicinity of the critical temperature, when QPs and HFP are in quasi-thermal equilibrium. Assuming that the energy gap is more or less isotropic, the temperature dependence of the photo-excited QP density $n_{\mathrm{pe}}$ (which corresponds to $\frac{\eta}{R}\left[q(\infty)-q_{T}\right]$ discussed in Sect. 2.1.1) is given by:

$$
n_{\mathrm{pe}}=\frac{\mathcal{A} /\left(\Delta(T)+k_{B} T / 2\right)}{1+\frac{2 v}{N(0) \hbar \Omega_{c}} \sqrt{\frac{2 k_{B} T}{\pi \Delta(T)}} \exp \left(-\Delta(T) / k_{B} T\right)},
$$

where $\hbar \Omega_{c}$ is the phonon cut-off energy and $v$ the effective number of phonon modes per unit cell (or phonon branches) participating in the relaxation. Using this expression, and considering the BCS dependence of the superconducting gap $\Delta(T)$, this expression is often used to determine the gap magnitude even for systems, where $\Delta(T \rightarrow 0)$ may not be spectroscopically determined. Examples of such analysis are studies of the superconducting gap in cuprates [14, 50, 98-102] (see also Sect. 2.2), pnictides [54, 55, 103] and organic superconductors [104]. Furthermore, a similar expression [15] has also been derived for the case where the energy gap is temperature independent, e.g. when discussing pseudo-gaps in cuprate superconductors [14, 50, 98-102].

\subsection{Superconducting Gap Dynamics in High-T Cuprate Superconductors}

In the above sections we focused on the real-time dynamics in conventional BCS superconductors with s-wave (isotropic) gap. Cuprate high- $T_{c}$ superconductors, however, are characterized by an anisotropic d-wave gap with nodes (directions in the momentum space with vanishing gap). Thus, one may expect their behavior under non-equilibrium conditions may be substantially different. Surprisingly, however, there seem to be more similarities than differences between the two classes of superconductors in terms of dynamics. Instead of a detailed review of the field of dynamics in cuprate superconductors, which can be found in Ref. [23], we briefly review the similarities and anomalies with respect to the observations in conventional BCS s-wave superconductors (discussed in sections above). 
Figure 10a presents the typical data obtained on cuprate superconductors in the low excitation density limit. It shows the temperature dependence of photo-induced reflectivity $(\Delta R / R)$ traces on $\mathrm{La}_{1.9} \mathrm{Sr}_{0.1} \mathrm{CuO}_{4}$ single crystal $\left(T_{c}=30 \mathrm{~K}\right)$ [99]. The data are often described in terms of a two-component response, with component A (see Fig. 10a) present only below $T_{c}$. Component B, however, is present to temperatures far above $T_{c}$. Probe polarization [98, 102] and chemical doping [14, 99] dependent studies revealed the coexistence of the two components below $T_{c}$. On the other hand, the correlation of the (doping-dependent) onset temperature of the component B $[14,99]$ has been made with the so called "pseudo-gap temperatures" determined by other methods, sensitive to the changes in the low energy density of states [105]. These suggest the origin of the component B being in the response of the competing order, like, e.g. (fluctuating) charge stripes or density waves.

From here on, we focus on the component (A) that can be clearly linked to the dynamics of the superconducting condensate, as demonstrated in Fig. 11. Here, a comparison of the dynamics, recorded by (a) probing the changes in reflectivity at $800 \mathrm{~nm}$ and (b) the $\mathrm{THz}$ conductivity as a function of excitation density $(\mathrm{T}=4 \mathrm{~K})$ is presented. At low excitation densities, where the fast relaxing component (B) associated to the dynamics of the competing order is small compared to the response of the condensate, both responses (reflectivity changes and changes in the $\mathrm{THz}$ conductivity) are comparable. Only for high excitation densities, when superconductivity becomes strongly suppressed, i.e. when the photoinduced change is saturating, the difference between the two responses becomes obvious. We note that the fast relaxing component $\mathrm{B}$ is absent (or dramatically reduced) in the $\mathrm{THz}$ response. The reason for that lies in the nature of the $\mathrm{THz}$ response of a superconductor, which is governed by the change of the phase of the transmitted $\mathrm{THz}$ electric field pulse
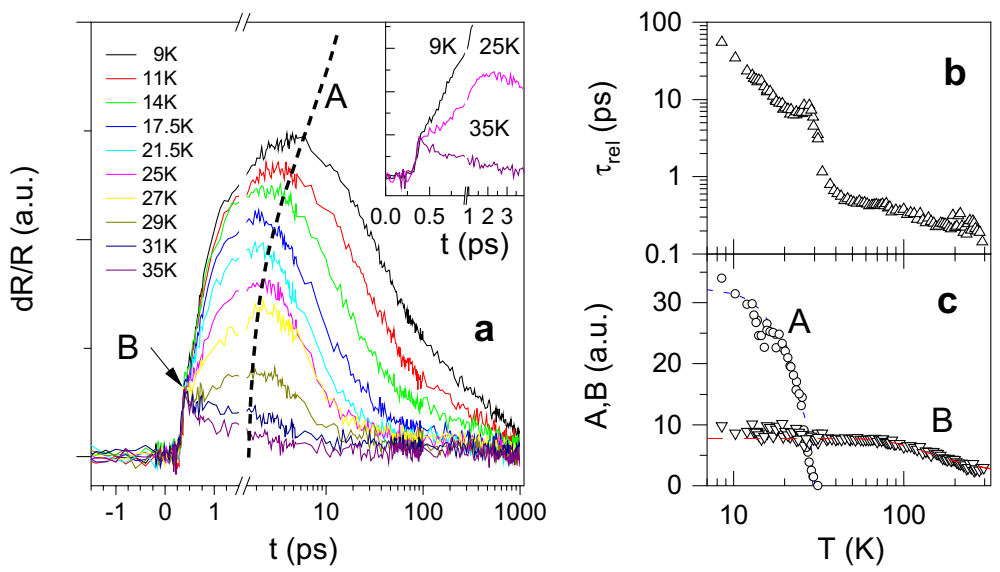

Fig. 10 a Dynamics of photo-induced change in reflectivity $\Delta R / R$ in $\mathrm{La}_{1.9} \mathrm{Sr}_{0.1} \mathrm{CuO}_{4}$ single crystal $\left(T_{c}=30 \mathrm{~K}\right)$ recorded in the low excitation density limit. Two distinct components (A and B) are observed when probing dynamics with near-infrared pulses (see also inset). b The temperature dependence of relaxation time of the reflectivity signal. $\mathbf{c}$ The temperature dependence of amplitudes of the two components, including fits with the model by Kabanov et al. [15]. For details, see the original publication [99], from which the data are reproduced (Color figure online) 

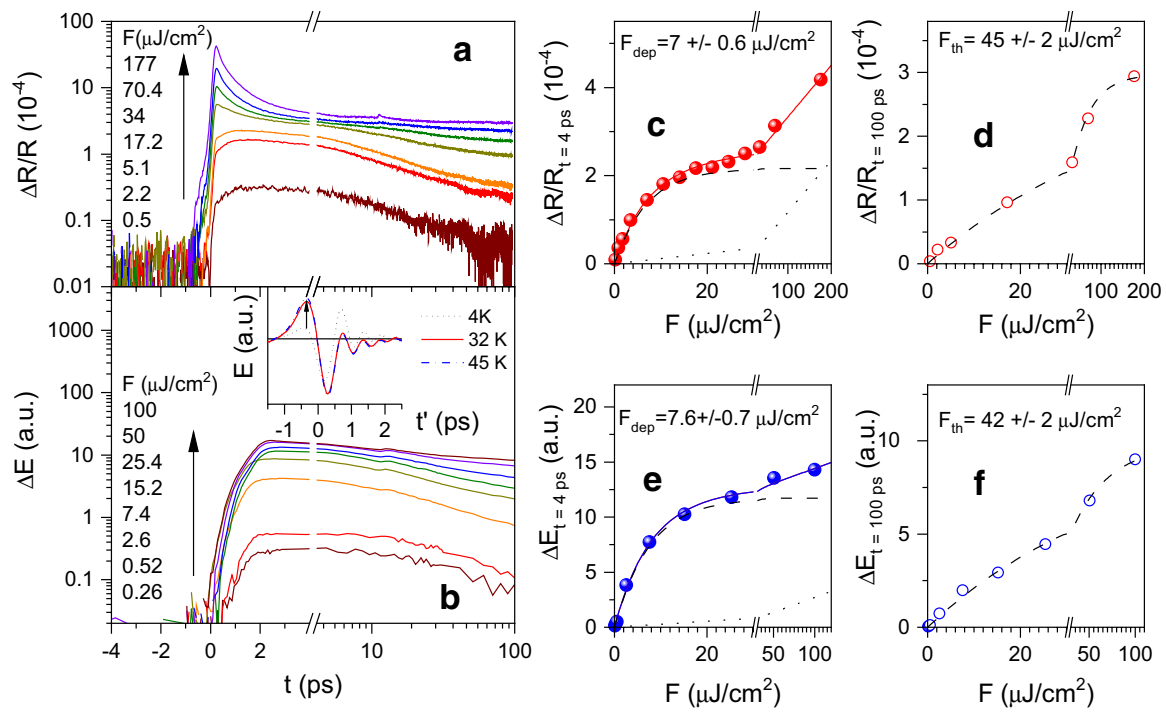

Fig. 11 Excitation density dependence of dynamics in $\mathrm{La}_{1.84} \mathrm{Sr}_{0.16} \mathrm{CuO}_{4}$ thin film $\left(T_{c}=31 \mathrm{~K}\right)$ at $4 \mathrm{~K}$, excited by $50 \mathrm{fs}$ near-infrared $(800 \mathrm{~nm})$ pulses. The dynamics are probed in two different experimental configurations: a by recording changes in reflectivity at $800 \mathrm{~nm}$ and $\mathbf{b}$ by probing changes in $\mathrm{THz}$ conductivity (spectrally integrated approach). c, $\mathbf{d}$ present the excitation dependence of reflectivity change at $\mathbf{c} 4 \mathrm{ps}$ and $\mathbf{d} 100 \mathrm{ps}$ after photo-excitation. The corresponding amplitudes of the change in the electric field at a fixed time delay (see inset to $\mathbf{b}$ ) at the two distinct time delays are shown in $\mathbf{e}$ and $\mathbf{f}$, respectively. Note that the THz electric field is mainly sensitive to superconductivity, as exemplified in inset to b. At long time delays ( $100 \mathrm{ps}$ ) the system is already thermalized, and the corresponding response is a bolometric one. Indeed, the values for characteristic fluence require to heat up the film to above $T_{c}, F_{t h}$, perfectly match the thermodynamic calculation [60]. In $\mathbf{d}, \mathbf{f}$ the presented data are taken from Ref. [60] (Color figure online)

(caused by the inductive character of the complex optical conductivity in the superconducting state)—-see inset to Fig. $11 \mathrm{~b}$.

Now, let us address the similarities (and some surprising inconsistencies) of the condensate dynamics in high- $T_{c}$ cuprates with conventional superconductors.

\subsubsection{Pair-Breaking Dynamics in Cuprates}

Looking at Figs. 10a and 11a, b, one realizes a similar delayed pair-breaking dynamics as in $\mathrm{NbN}$ and $\mathrm{MgB}_{2}$ shown in Fig. 4. While the timescale is substantially shorter (depends also on the compound), one can argue in favor of pair-breaking by absorption of bosonic excitations created during the avalanche relaxation of hot QPs. This result suggests the same type of boson-bottleneck governing the relaxation as in conventional superconductors. Here, considering the possible scenarios of superconductivity in cuprates being mediated by phonons or magnetic excitations, we cannot specify a priori which bosonic excitation may be responsible for pair-breaking (or pairing). 
We should further mention the study on $\mathrm{YBa}_{2} \mathrm{Cu}_{3} \mathrm{O}_{7}$ crystals, where the dynamics of both, the condensate and specific IR-active $c$ axis phonons were studied using time-resolved broadband $\mathrm{THz}$ conductivity [59]. The dynamics in the superconducting state is presented in Fig. 12. Panel (a) presents the in-plane response, showing a rapid filling-in of the superconducting gap (equilibrium $\sigma_{1}(\omega)$ in the superconducting and normal state are shows at the top) and its recovery on the timescale of a few ps. The dynamics is similar to the results of earlier studies using near-infrared probe [14] of 0.2-1 THz pulses [17]. Panel (b) presents the out-of-plane ( $c$ axis) response, involving the dynamics of two $c$ axis polarized phonons. Especially the apex oxygen mode at $\approx 17 \mathrm{THz}$ is found to be strongly affected by superconductivity, giving rise to a highly asymmetric line-shape in the superconducting state. The analysis of the time-evolution of the phonon line-shape in panel (c) provides access to the dynamics of the condensate, related to the phonon asymmetry parameter shown in (d), and the phonon frequency. The later, presented in panel (e), displays a rapid red-shift of the mode and its sub-picosecond recovery. Since the red-shift is characteristic of an anharmonic lattice potential, the shift of the apex phonon frequency can be used as a probe of the vibrational occupation. Indeed, the presented frequency shift in Fig. 12e suggests a rapid heating of the mode, reaching occupations compared to those achieved by heating up to $200 \mathrm{~K}$ [59]. The results indicate highly excited lattice modes on the timescale comparable to pair-breaking.

\subsubsection{Superconducting State Recovery Dynamics in Cuprates}

The relaxation time in many of the cuprate superconductors shows similar temperature $[50,83,99,106]$ (see also Fig. 10b) and excitation density dependence [58, 61,83 ] as observed in $\mathrm{NbN}$ [22] and predicted by the Rothwarf-Taylor model for
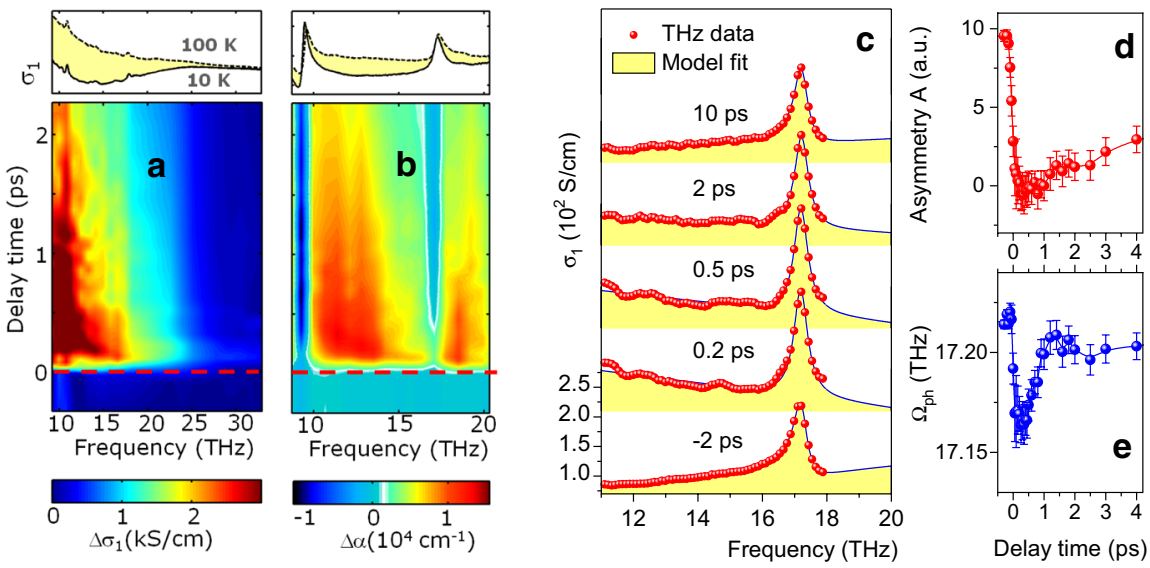

Fig. 12 Spectrally resolved studies of the broadband $\mathrm{THz}$ conductivity in $\mathrm{YBa}_{2} \mathrm{Cu}_{3} \mathrm{O}_{7}$ at $20 \mathrm{~K}$ [59]. a Inplane response, showing photo-induced filling of the gap and its subsequent recovery on the ps timescale. b $c$ axis response, capturing the dynamics of $2 c$ axis phonons. c The line-shape analysis of the apex oxygen mode at 17.2 THz [59]. The time-evolution of the two relevant parameters, the asymmetry parameter d and the mode central frequency e. Data are reproduced from Ref. [59] (Color figure online) 
both weak and strong bottleneck cases [78]. Together with the observed delayed pair-breaking, discussed above, the data seem to be generally consistent with the strong boson-bottleneck scenario. On the other hand, some doping-dependent studies of carrier relaxation dynamics suggest that the character of relaxation may change between the under-doped and the over-doped regions of the phase diagram. In particular, Gedik et al. [52] observed that the relaxation time is temperature and excitation density dependent in the under-doped case, while in over-doped at low temperatures the gap recovery dynamics is independent on excitation density and temperature. Indeed, the observation in $\mathrm{Bi}_{2} \mathrm{Sr}_{2} \mathrm{Ca}_{1-y} \mathrm{Dy}_{y} \mathrm{Cu}_{2} \mathrm{O}_{8}$ series [52] is consistent with the observation on $\mathrm{YBa}_{2} \mathrm{Cu}_{3} \mathrm{O}_{7-\delta}$ samples, where in the over-doped range the relaxation dynamics at low temperatures seems to be independent on temperature and excitation density [14]. On the other hand, systematic doping dependence studies on single-layer cuprates do not seem to show this disparity $[99,106]$.

\subsubsection{Energetics of the Gap Suppression in Cuprate Superconductors}

There are two important aspects of dynamics in cuprates that are revealed by studying the excitation density dependent suppression of superconductivity.

First of all, in the low excitation density limit, the photo-induced changes in the QP density in cuprates scale linearly with the excitation density. Such an observation is straightforward for the case of a superconductor with an isotropic gap. Here the number of broken pairs per absorbed photon with energy $\hbar \omega$ is simply given by $n_{\mathrm{pe}}=\hbar \omega / 2 \Delta$ (or a fraction thereof-see Sect. 2.1.1). But, for an anisotropic gap with nodes this observation is unexpected [15]. Here, the quasiparticle density of states for energies $\varepsilon \ll \Delta_{a}$, where $\Delta_{a}$ is the maximal gap in the anti-nodal direction, is given by $N(\varepsilon)=N(0)\left(\frac{\varepsilon}{\Delta_{a}}\right)^{\kappa}$ (power $\kappa$ depends on the topology of the nodes on the Fermi surface). Therefore, at low temperatures, a sub-linear behavior of the photo-induced QP density with excitation intensity is expected [15], with $n_{\mathrm{pe}} \propto A^{(\kappa+1) /(\kappa+2)}$. For quasi $2 \mathrm{D}$ cuprates, with $\kappa=1$, this would suggest $n_{\mathrm{pe}} \propto A^{2 / 3}$ dependence at low temperatures, which was never observed.

In fact, even the temperature dependence of $n_{\mathrm{pe}}$ extracted from time-resolved experiments, seems to be at odds with the behavior expected for a d-wave superconductor $[15,107]$. A possible origin of this apparent contradiction may be in a slow relaxation of hot QPs towards the nodes due to kinematic constraints (i.e. the energy and momentum conservation) [108].

Another interesting aspect related to the excitation dependent studies of suppression of superconductivity in cuprates is related to the energy required to deplete superconductivity. As shown in Sect. 2.1.2, the absorbed energy density required to suppress superconductivity roughly matches the condensation energy in $\mathrm{NbN}$ [22]. Systematic studies on cuprates $[52,60,61]$, however, show that the energy required to suppress superconductivity exceeds the condensation energy by about one order of magnitude. Figure 11 presents the fluence dependence of the superconducting state depletion in $\mathrm{La}_{1.84} \mathrm{Sr}_{0.16} \mathrm{CuO}_{4}$ using near-infrared pump pulses in two configurations. The saturation of the photo-induced change in reflectivity or $\mathrm{THz}$ conductivity at 4 ps time delay is observed at the fluence $\approx 7 \mu \mathrm{J} / \mathrm{cm}^{2}$, which corresponds to 
the absorbed energy density of $2.4 \mathrm{k}_{B} \mathrm{~K}$ per $\mathrm{Cu}$ atom. This energy is about 8 times higher than the condensation energy $\approx 0.3 \mathrm{~K} / \mathrm{Cu}$ [109]. One possible reason for this discrepancy is a large magnitude of the superconducting gap in $\mathrm{La}_{2-x} \mathrm{Sr}_{x} \mathrm{CuO}_{4}$, compared to the Debye energy. In other words, in $\mathrm{La}_{2-x} \mathrm{Sr}_{x} \mathrm{CuO}_{4}$ there are several optical phonon branches with $\hbar \omega<2 \Delta_{a}$ ( $\Delta_{a}$ is the gap energy in anti-nodal direction), thus a large amount of the absorbed optical energy can be transferred to phonons with $\hbar \omega$ $<2 \Delta_{a}$, on the $100 \mathrm{fs}$ timescale. Since pair-breaking by phonons with $\hbar \omega<2 \Delta_{a}$ is strongly suppressed, these could serve as an energy sink during the initial avalanche relaxation of hot carriers towards the gap edge.

Recently, a similar study in the electron-doped cuprate superconductor $\mathrm{Pr}_{2-x} \mathrm{Ce}_{x} \mathrm{CuO}_{4}$ was performed [61]. Here melting of the superconducting state with both near-infrared and $\mathrm{THz}$ pulses was studied. Using THz pulses, tuned just above the gap, the absorbed energy density required to deplete SC was found to match the condensation energy. With near-infrared excitation, however, the absorbed energy density was again a factor of 5-6 higher than the condensation energy-despite the fact that in $\mathrm{Pr}_{2-x} \mathrm{Ce}_{x} \mathrm{CuO}_{4}$ the gap, $2 \Delta$, is small compared to all relevant bosonic energy scales [61]. The data imply that following optical pumping a rapid electron-boson energy transfer takes place, yet only selected bosonic modes (e.g. antiferromagnetic fluctuations, or specific lattice modes) couple to the condensate. Further systematic studies, with selective (tuned) pumping are clearly required here.

\subsubsection{Open Questions}

Most of the observations obtained by systematic temperature and excitation dependent studies of the dynamics in the superconducting state show great similarities between cuprate superconductors and conventional BCS superconductors. Both pair-breaking and the superconducting state recovery dynamics are found to be consistent with a boson-mediated pairing in cuprates. The nature of the pairing "glue" is, however, still an open question. On the other hand, unlike in BCS superconductors, photoexcitation does not seem to give rise to a reduction of the gap magnitude (as seen for $\mathrm{NbN}$ in Fig. 3), but rather to filling-up of the gap, i.e. to an increase of the spectral weight inside the gap [68].

\section{Enhancement of Superconductivity by Resonant Excitation}

\subsection{THz Driven Enhancement of Superconductivity in BCS Superconductors}

In Sect. 1.2 we addressed the early studies of enhancement of superconductivity with sub-gap excitation. Most of these experiments were conducted using continuous excitation with sub-gap radiation, and the effects (increase of the superconducting gap, critical current and transition temperature) were typically of the order of $1 \%$, limited by the QP scattering rate and the radiation induced sample heating. Using the novel pulsed sources of intense infrared radiation one may expect to observe transient effects, whose magnitude would exceed magnitudes observed in early studies. Here we briefly review a study, which suggest superconductivity enhancement 
effects in a dirty-limit superconductor $\mathrm{NbN}$ [110] on a 100 ps timescale, when the system is driven by narrow-band $\mathrm{THz}$ pulses tuned close to the gap energy.

In the study, summarized in Fig. 13 , narrow band THz pulses $(\approx 30 \mathrm{GHz}$ bandwidth, 20 ps duration) were used to both excite $\mathrm{NbN}$ and probe the resulting changes in the transmission. The studies using near-infrared excitation [22] demonstrated that the time evolution of the $\mathrm{NbN}$ superconductor can be, for time delays beyond the pair-breaking process, described by the time evolution of effective temperature $T^{*}$ of thermalized condensate, QPs and HFPs. As the transmission through the sample is strongly affected by the opening of the superconducting gap, one has-in principle-access to the time evolution of the gap, without having spectral resolution. With these assumption, two types of experiments were performed: above gap pumping at $2.08 \mathrm{THz}$, with traces shown in panel (a), and pumping with $1.26 \mathrm{THz}$ pulses (which is below $2 \Delta$ for $T<11 \mathrm{~K}$-see inset to Fig. 13a) with traces at different temperatures shown in panels (c1)-(c6).

Two interesting observations were made, both suggesting an additional mechanism present at temperatures close to $T_{c}$, competing with the expected light induced suppression of superconductivity.

In experiments with above-gap excitation, the absorbed energy density required to deplete superconductivity, $A_{\text {dep }}$, was determined at different base temperatures, similar to experiments performed with near-infrared pumping (see Fig. 6). The surprising observation is that $A_{\text {dep }}$ is increasing with increasing temperature (Fig. 13b) opposite to the experiments with near-infrared $(1.55 \mathrm{eV})$ pumping. This is a first
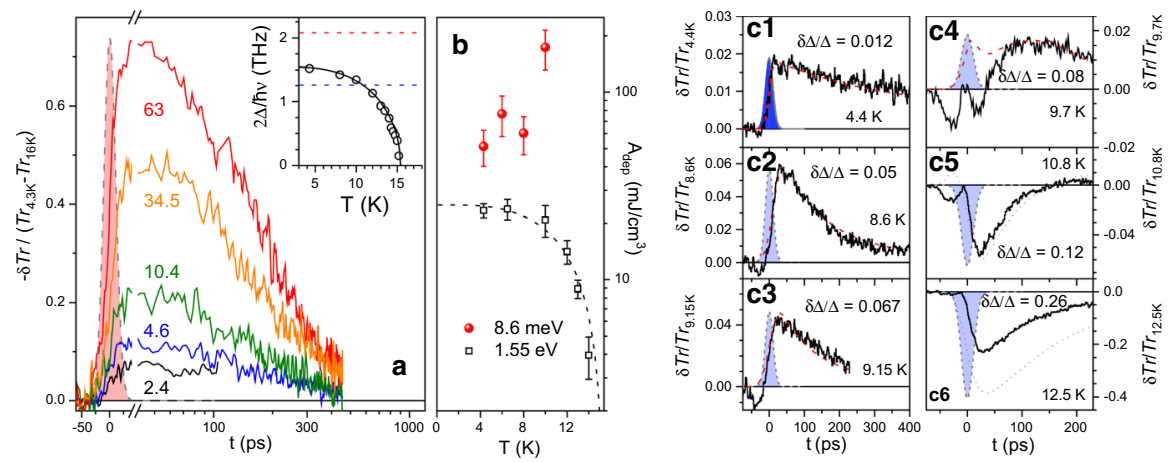

Fig. 13 Dynamics in $\mathrm{NbN}$ films driven by narrow-band $\mathrm{THz}$ excitation [110]. a Dynamics of the relative change in transmission $\delta \operatorname{Tr}$ (normalized to $\operatorname{Tr}_{4.3 K^{-}} \operatorname{Tr}_{16 K}$ ) at $4.3 \mathrm{~K}$ when pumping with $2.08 \mathrm{THz}$ pulses of different absorbed energy density $A$ (in $\mathrm{mJ} / \mathrm{cm}^{3}$ ). The shaded region presents the pump-probe intensity auto-correlation. Inset shows the T-dependence of gap with the two frequencies used in narrow band THz pump-probe experiments by the dashed lines. b Comparison of the absorbed energy density required to deplete superconductivity, measured with near-infrared pumping at $1.55 \mathrm{eV}$ [22] and $\mathrm{THz}$ pumping at $8.3 \mathrm{meV}(2.08 \mathrm{THz})$. The dashed line represents the condensation energy. (c1-c6) Recorded traces of differential transmission for pumping at $1.26 \mathrm{THz}$ at different temperatures (black solid lines). The shaded region presents the pump-probe intensity auto-correlation. For red dashed lines present the behavior in the case of the so called $T^{*}$-model, which adequately described the dynamics for near-infrared excitation [22]. At each temperature the maximum relative change in the gap $\delta \Delta / \Delta$ is estimated from the measured $\delta \operatorname{Tr} / \operatorname{Tr}$ (Color figure online) 
indication that an additional process may be competing with the common lightdriven suppression of superconductivity.

Even stronger evidence of a competing process is provided by pumping at 1.26 THz. Panels (c1)-(c6) present the recorded traces of the relative transmission change, compared to the simulations assuming the $T^{*}$ model, i.e. the assumption that the superconducting state at $\sim 30$ ps after photo-excitation can be described by $\Delta^{*}=\Delta\left(T^{*}\right)$. The simulations are given by the dashed red and dotted blue lines. The non-monotonous behavior is a result of the fact that at $1.26 \mathrm{THz}$ the transmission is a non-monotonous function of temperature [110]. For time delays less than $\approx 50$ ps, the relative transmission changes systematically shows the opposite direction to the one predicted by the $T^{*}$-model. This is especially pronounced for near-resonant pumping, when $2 \Delta / h \approx 1.26 \mathrm{THz}$, around $10 \mathrm{~K}$. This observation suggests a transient gap enhancement (and the corresponding increase in condensate density) under $\mathrm{THz}$ pumping for timescales less than $\approx 50 \mathrm{ps}$.

Given the fact that these effects are pronounced in the vicinity of critical temperature, when the density of thermally excited QPs becomes prominent, the data are interpreted within the Eliashberg scenario [36, 110]. Here, narrow-band excitation gives rise to a highly non-thermal QP distribution resulting in amplification of SC that would be washed out under continuous excitation. Taking the data at face value, the enhancement effects seem to be observed even in the case of above gap excitation, where QP excitation competes with pair-breaking. One could imagine that such experiments, if performed with pulses at even lower carrier frequencies and closer to the critical temperature, may even lead to stronger effects as discussed above.

\subsubsection{Photo-Induced Superconductivity in Unconventional Superconductors}

Following the ideas of the pioneering works on radiation-enhanced superconductivity addressed in Sect. 1.2, we discussed first experiments utilizing intense narrow band pulses, aiming to transiently enhance superconductivity in conventional BCS superconductors. We should, however, also briefly address more recent studies reporting superconducting-like transient states (with the typical duration of $1 \mathrm{ps)}$ observed in cuprate superconductors [111-114], fullerides [115-118] and recently also on organic charge-transfer salts [119] when photoexciting at base temperatures far above the corresponding equilibrium critical temperatures in these systems. The leading idea in these experiments is to excite specific lattice modes/distortions by utilizing nonlinear phononics $[120,121]$. This way, pulsed excitation can either suppress a competing (stripe) order $[111,122]$, or induce superconductivity by the lattice-driven modification of the pairing interaction [111-115].

Figure 14 presents the experimental approach and the results obtained in one of the first studies of this type, the study of light-induced melting of the competing stripe order in $\mathrm{La}_{1.675} \mathrm{Eu}_{0.2} \mathrm{Sr}_{0.125} \mathrm{CuO}_{4}\left(\mathrm{LESCO}_{1 / 8}\right)$ [111]. The non-superconducting $\mathrm{LESCO}_{1 / 8}$ is known to possess a static stripe order. Upon excitation with infrared pulses, the induced changes in reflectance (Fig. 14c) display a shoulder-like structure around $60 \mathrm{~cm}^{-1}$, which was interpreted as a photoinduced $c$ axis Josephson plasma resonance (JPR) [111]. The JPR is a general feature of layered cuprate superconductors, explained by the Josephson coupling between the adjacent superconducting 

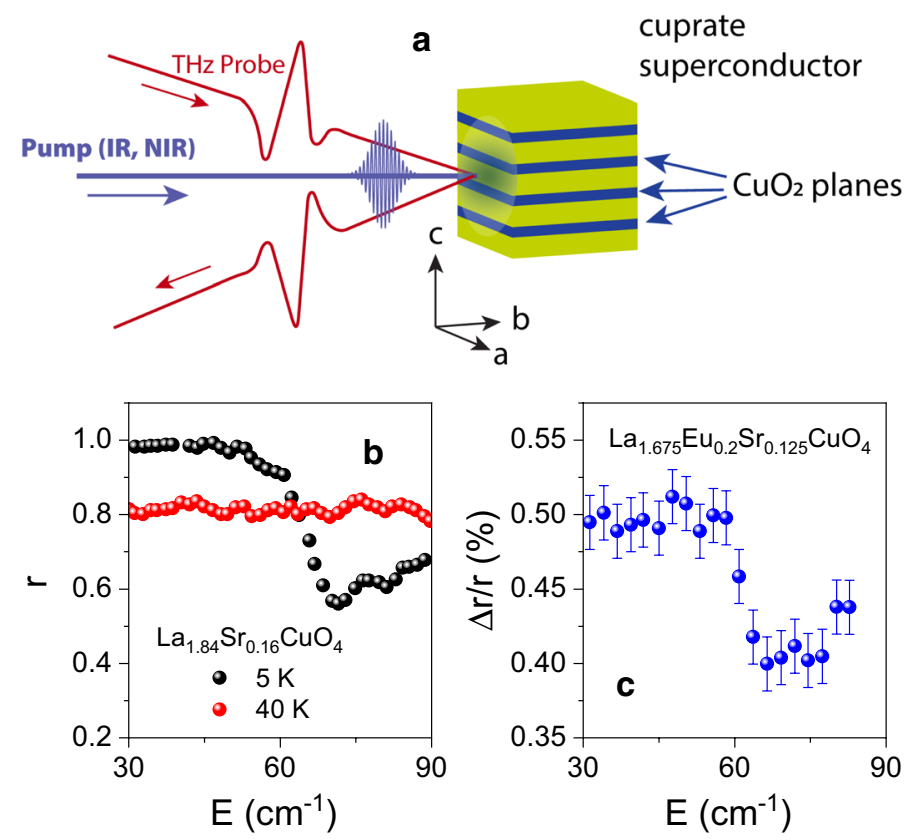

Fig. 14 Studies of photoinduced superconductivity in cuprate superconductors. a A typical experimental configuration, probing the induced changes in the out-of-plane ( $c$ axis) $\mathrm{THz}$ conductivity following mid-infrared (or near-infrared) excitation. b The changes in equilibrium $c$ axis reflectance $\left(r=E_{\text {refl }} / E_{\text {inc }}\right)$ upon cooling the optimally doped LSCO $\left(\mathrm{La}_{1.84} \mathrm{Sr}_{0.16} \mathrm{CuO}_{4}\right)$ through $T_{c}=38 \mathrm{~K}$ (adapted from Ref. [111]). c The photoinduced change in reflectance in stripe-ordered (non-superconducting) $\mathrm{La}_{1.675} \mathrm{Eu}_{0.2} \mathrm{Sr}_{0.125} \mathrm{CuO}_{4}$ after photoexcitation with infrared pulse at $16 \mu \mathrm{m}$ (adapted from Ref. [111]) While the overall reflectance change is positive in the $\mathrm{THz}$ spectral range, the part of the response having a step-like shape was attributed to the light induced superconductivity (Color figure online)

$\left(\mathrm{CuO}_{2}\right)$ layers and can be observed in equilibrium studies of $c$ axis optical conductivity, as shown in Fig. 14b. The fact that the induced changes in reflectance, $\Delta r / r$, are small (the step in $\Delta r / r$, attributed to the JPR is only about $0.1 \%$, to be compared to the $\approx 40$ and the reflectivity of $30 \%$ effect in the equilibrium), could be attributed to a large mismatch of the penetration depths. Indeed, the pump field penetration depth at $16 \mu \mathrm{m}$ is about $200 \mathrm{~nm}$ while that of the $\mathrm{THz}$ probe field is about $10 \mu \mathrm{m}$ [111]. The comparably large, frequency independent offset in $\Delta r / r$ was however attributed to the photoinduced changes in the high(er) frequency phonons [111]. By processing the raw data, taking into account the large penetration depth mismatch and subtracting a frequency independent component originating from perturbed higher-frequency modes, the authors showed that $\sigma_{2}$ from the photo-excited layer displayed the upturn at low frequencies, resembling the response of the superconductor [111]. The fact that the effect was peaked around the pump-photon energy of $80 \mathrm{meV}(15 \mu \mathrm{m})$ leads to the interpretation of photoinduced superconductivity via melting of the underlying static stripe order [111]. The result is particularly surprising, considering the excitation densities in these studies are of the order of $1 \mathrm{~mJ} / \mathrm{cm}^{2}$, 
nearly two orders of magnitude higher than excitation densities required to suppress superconductivity in an optimally doped LSCO, when excited by NIR pulses polarized in the $a-b$ plane (see Fig. 11).

Following this pioneering work, the approach has been extended to the superconducting cuprates. Using the same approach, it was shown that the transient state with the low-frequency divergence in $\sigma_{2}(\omega)$, reminiscent of $1 / \omega$ inductive response of the condensate, could be realized over large range of doping and temperatures, approaching room temperature in strongly underdoped cuprates $[112,114,121]$. The interpretation of these data is, however, still under intense discussion, ranging from aspects of the data analysis to the different interpretations of the origin of the lightinduced modifications of low frequency $\sigma(\omega)$ [123-131]. Recently, several groups performed similar studies of the $c$ axis THz response using both mid-infrared as well as near-infrared pulses for photoexcitation [122, 125, 126, 132-134]. While it was demonstrated that near-infrared excitation results in similar changes in the low-frequency conductivity as with mid-infrared excitation [132, 134], the consensus on the nature of the photoinduced state is still lacking. In the superconducting state of the underdoped LSCO Niwa et al. [125] observed a photoinduced red shift of the JPR, consistent with the photoinduced suppression of superconductivity. It was argued that transient heating linked to the inhomogeneous excitation profile could give rise to potential artefacts in the analysis [125]. Studies in the superconducting state of $\mathrm{La}_{1.905} \mathrm{Ba}_{0.095} \mathrm{CuO}_{4}$ [132], $\mathrm{La}_{1.885} \mathrm{Ba}_{0.115} \mathrm{CuO}_{4}$ [122], and $\mathrm{Pr}_{0.88} \mathrm{LaCe}_{0.12} \mathrm{CuO}_{4}$ [133], however reported a photoinduced red shift of the JPR and the appearance of an additional mode at higher frequencies. While at temperatures above $T_{c}$ no such photoinduced mode was observed in $\mathrm{La}_{1.905} \mathrm{Ba}_{0.095} \mathrm{CuO}_{4}$ [132], it has been observed in $\mathrm{La}_{1.885} \mathrm{Ba}_{0.115} \mathrm{CuO}_{4}$, albeit at very high excitation density of $9 \mathrm{~mJ} / \mathrm{cm}^{2}$ [122]. Finally, following up on the first reports, suggesting signatures of transient (inhomogeneous) superconductivity up to room temperature in underdoped $\mathrm{YBa}_{2} \mathrm{Cu}_{3} \mathrm{O}_{6+x}[112,114]$, similar studies have been performed by both mid-infrared and near-infrared excitation [126]. While, similarly to earlier reports $[112,114]$, a photoinduced increase of $\mathrm{THz}$ reflectivity was observed, the authors argue against the photoinduced (inhomogeneous) superconductivity [126]. From the above, it is clear that further systematic studies are required. Ideally, the experiments should be performed on $a$ axis oriented thin films in transmission geometry, to insure homogeneous excitation profile, and to enhance the experimental sensitivity (as shown in Sect. 2, superconductivity results in vehement changes in thin film transmission).

We note that similar studies to those in cuprates were performed also on $\mathrm{K}_{3} \mathrm{C}_{60}$ [115-118] and recently also on the organic charge-transfer salt $\kappa$-(BEDT$\mathrm{TTF})_{2} \mathrm{Cu}\left[\mathrm{N}(\mathrm{CN})_{2}\right] \mathrm{Br}$ [119]. In both materials, upon photoexcitation with intense mid-infrared pulses, spectral signatures of superconductivity were reported for base temperatures well above the corresponding equilibrium critical temperatures. In $\mathrm{K}_{3} \mathrm{C}_{60}$ the mismatch between the pump and the probe penetration depths was still large [115], requiring multilayer modelling (as in cuprates). This does not seem to be the case for the organic charge-transfer salt [119]. At face value, the extracted optical conductivities in the transient states indeed seem like to result from photoinduced superconductivity, i.e. showing gapping (reduction) of $\sigma_{1}$ and $\sigma_{2}(\omega) \propto 1 / \omega$. In $\mathrm{K}_{3} \mathrm{C}_{60}$, where the recent study suggests the photoinduced state to be long-lived 
with lifetime well in the nanosecond range [118], the optical conductivity data is supplemented by the DC transport studies, demonstrating the photoinduced drop in resistivity [118].

Despite the apparent agreement with the proposed scenario of light-enhanced/ induced superconductivity, where mid-infrared pulses induce large-amplitude structural distortions and thereby modulate the pairing strength $[115,118]$, there are still several open questions. For example, experiments on $\mathrm{K}_{3} \mathrm{C}_{60}$ performed at temperatures below $T_{c}$ show a photoinduced suppression of superconductivity [115] instead of the expected gap enhancement. Apart from the proposed exciton-cooling model [129] this observation seems at odds with the majority of the proposed scenarios [115, 127, 135-137]. Secondly, the excitation densities in most of these experiments are extremely high. For example, the recent study reporting the generation of a long-lived (nanoseconds) metastable superconducting state in $\mathrm{K}_{3} \mathrm{C}_{60}$ [118] suggests a fluence threshold for the effect of about $20 \mathrm{~mJ} / \mathrm{cm}^{2}$. With the pump penetration depth of $200 \mathrm{~nm}$, and the reflectivity of $30 \%$ [115, 118] one obtains the absorbed energy density of the order of $700 \mathrm{~J} / \mathrm{cm}^{3}$. The resulting temperature of the excited volume, assuming the system is quasi-thermalized on the $1 \mathrm{~ns}$ timescale, can be estimated from the temperature dependence of the total specific heat of $\mathrm{K}_{3} \mathrm{C}_{60}$ [138], and amounts to about $500 \mathrm{~K}$ ! Here we should mention a recently proposed alternative scenario, where the experimental data are accounted for by considering that the pulse, resonant with a phonon mode, creates a (nonsuperconducting) nonequilibrium state in which the linear response conductivity becomes negative [130,131].

\section{Summary and Perspectives}

In this brief review, we addressed some aspect of superconductors, driven out of equilibrium using femtosecond and picosecond optical pulses. The real-time experimental approaches and theoretical models presented here, building on the pioneering works from the early era of non-equilibrium superconductivity (Sects. 1.1, 1.2), provide new insights into this fascinating field of condensed matter physics.

This review is, however, far from comprehensive in view of addressing all aspects related to light-driven out-of-equilibrium phenomena in superconductors. We briefly discussed novel approaches, aiming to impulsively modulate the pairing strength and thereby enhance or induce superconductivity at temperatures far above the superconducting critical temperature (Sect. 3.1.1). In addition, we should also mention the recent efforts in using intense $\mathrm{THz}$ pulses to probe collective modes of superconductors (for classification of collective modes in superconductors see, e.g. [139]).

The amplitude mode of the superconducting order parameter, describing variations of the amplitude of the order parameter with the frequency of $2 \Delta / h$, commonly referred to as the Higgs mode, is a scalar excitation of the order parameter [140]. As it does not carry spin or charge, the Higgs mode does not couple linearly to the electromagnetic field. Only when superconductivity coexists with a charge density wave (CDW) order, as in $2 \mathrm{H}-\mathrm{NbSe}_{2}$ [141-143], has Higgs mode been detected using linear spectroscopy $[141,143]$. In this case, the amplitude mode of the CDW order 
couples to the Higgs mode via modulation of the density of states at the Fermi level and thereby perturbing the superconducting condensate.

There were numerous theoretical reports, suggesting that the excitation of Higgs mode could be realized by quenching the superconducting order [144] on a timescale shorter than the mode frequency $[145,146]$. These were followed by experimental studies using intense $\mathrm{THz}$ pulses, utilizing the $\mathrm{THz}$ pump- $\mathrm{THz}$ probe approach [147, 148] and the third harmonic generation [148, 149]. These results suggest the Higgs mode to be observed through its nonlinear coupling to the intense $\mathrm{THz}$ light fields. On the other hand, it has been argued that the third harmonic generation (THG) can also be attributed to the nonlinear optical excitation of collective lattice-charge fluctuations [150]. It has been argued that the THG signal due to density fluctuations should in fact dominate the response in a BCS superconductor [151]. While the question remains open [148, 151-153], we should mention yet another approach, where the Higgs mode is observed in the linear optical conductivity spectrum under injection of supercurrrent [154, 155]. Finally, in addition to the Higgs mode, unconventional superconductors with reduced dimensionality and multi-band superconductors may host a much richer spectrum of collective excitations $[139,156,157]$. The first experiments, suggesting the observation of the collective oscillation of the relative phase between the order parameters in a two-band superconductor $\mathrm{MgB}_{2}$, the Leggett mode, have just been reported [158].

Following the short summary, where we briefly introduced another field of studies of superconductors that has been made possible by the advancement in generation of intense electromagnetic pulses - the studies of the collective modes, we should reiterate some of the open questions and the possible ways of addressing them.

We have shown that the dynamics of the superconducting state of conventional BCS superconductors, excited by femtosecond optical pulses, can be extremely well described by the phenomenological Rothwarf-Taylor model [29, 78]. Surprisingly, the same can be said about experiments in the weak perturbation regime in unconventional d-wave superconductors, despite the fact that they are characterized by the anisotropic gap with nodes. Both pair-breaking and the superconducting state recovery dynamics are found to be qualitatively consistent with a boson-mediated pairing in cuprates. The question about the nature of the pairing "glue" is, however, still open. Here, systematic studies, by varying the energy of excitation photons, could provide further clues. Recent studies on the optimally doped PCCO suggest that pairing can be mediated either by bosons or antiferromagnetic fluctuations [61]. Thus, doping-dependent studies may be able to distinguish between the two scenarios.

Moreover, photoexcitation of cuprate superconductors seems to give rise to filling-up of the gap instead of the suppression of its magnitude [68]. Here a major progress in understanding can be expected from systematic tr-ARPES studies with high energy resolution and large momentum coverage [159-161].

Another rapidly developing field of research focuses on amplification of superconductivity. Here, studies in both conventional [110] and unconventional superconductors [111-114, 121, 122] require further investigations. In $\mathrm{NbN}$ [110], narrow band $\mathrm{THz}$ excitation studies suggest superconducting state enhancement to compete 
with pair-breaking, yet the spectrally resolved study is still lacking. Such studies could provide further evidence of non-thermal quasiparticle distribution, supporting the proposed scenario of Eliashberg enhancement of superconductivity [110]. Moreover, by tuning the excitation photon energy, detailed information of the underlying processes could be obtained, and compared to different theoretical proposals [41-42]. As far as the studies of photoinduced enhancement of superconductivity in unconventional superconductors are concerned, the main advancement could be expected by studying optically thin films. This way, one could insure a homogeneous excitation profile, and dramatically enhance the sensitivity.

Finally, in many of the unconventional superconductors, their ground state properties can be controlled by doping, pressure or strain. Here, a combination of pressure-tuning and impulsive excitation should provide a new playground to generate and investigate emergent (metastable) states, especially when pressure-tuning the materials into the vicinity of (quantum) critical points in their phase diagrams.

Acknowledgements Open Access funding provided by Projekt DEAL. The work was supported by the Deutsche Forschungsgemeinschaft (DFG, German Research Foundation)_TRR 173—26856537. We thank Viktor Kabanov for valuable discussions.

Open Access This article is licensed under a Creative Commons Attribution 4.0 International License, which permits use, sharing, adaptation, distribution and reproduction in any medium or format, as long as you give appropriate credit to the original author(s) and the source, provide a link to the Creative Commons licence, and indicate if changes were made. The images or other third party material in this article are included in the article's Creative Commons licence, unless indicated otherwise in a credit line to the material. If material is not included in the article's Creative Commons licence and your intended use is not permitted by statutory regulation or exceeds the permitted use, you will need to obtain permission directly from the copyright holder. To view a copy of this licence, visit http://creativecommons.org/licen ses/by/4.0/.

\section{References}

1. M. Tinkham, Introduction to Superconductivity: Chapter 11 (McGraw Hill, New York, 1996)

2. K.E. Gray (ed.), Nonequilibrium Superconductivity, Phonons and Kapitza Boundaries, NATO ASI Series (Plenum, New York, 1981)

3. R.E. Glover, M. Tinkham, Phys. Rev. 104, 844 (1956)

4. E. Burstein, D.N. Langenberg, B.N. Taylor, Phys. Rev. Lett. 6, 92 (1961)

5. A.F.G. Wyatt, V.M. Dmitriev, W.S. Moore, F.W. Sheard, Phys. Bevhav. Lett. 16, 1166 (1966)

6. A.H. Dayem, J.J. Wiegand, Phys. Rev. 155, 419 (1967)

7. T.M. Klapwijk, J.N. van den Bergh, J.E. Mooij, J. Low Temp. Phys. 26, 385 (1977)

8. T. Kommers, J. Clarke, Phys. Rev. Lett. 38, 1091 (1977)

9. J.F. Federici, B.I. Greene, P.N. Saeta, D.R. Dykaar, F. Sharifi, R.C. Dynes, Phys. Rev. B 46, 11153 (1992)

10. S.G. Han, Z.V. Vardeny, K.S. Wong, O.G. Symko, G. Koren, Phys. Rev. Lett. 65, 2708 (1990)

11. J.M. Chwalek, C. Uher, J.F. Whitaker, G.A. Morou, J.A. Agostinelli, Appl. Phys. Lett. 58, 980 (1991)

12. W. Albrecht, Th Kruse, H. Kurz, Phys. Rev. Lett. 69, 1451 (1992)

13. C.J. Stevens, D. Smith, C. Chen, J.F. Ryan, B. Podobnik, D. Mihailovic, G.A. Wagner, J.E. Evetts, Phys. Rev. Lett. 78, 2212 (1997)

14. J. Demsar, B. Podobnik, V.V. Kabanov, Th Wolf, D. Mihailovic, Phys. Rev. Lett. 82, 4918 (1999)

15. V.V. Kabanov, J. Demsar, B. Podobnik, D. Mihailovic, Phys. Rev. B 59, 1497 (1999)

16. R.A. Kaindl, M. Woerner, T. Elsaesser, D.C. Smith, J.F. Ryan, G.A. Farnan, M.P. McCurry, D.G. Walmsley, Science 287, 470 (2000) 
17. R.D. Averitt, G. Rodriguez, A.I. Lobad, J.L.W. Siders, S.A. Trugman, A.J. Taylor, Phys. Rev. B 63, 140502(R) (2001)

18. G.P. Segre, N. Gedik, J. Orenstein, D.A. Bonn, Ruixing Liang, W.N. Hardy, Phys. Rev. Lett. 88, 137001 (2002)

19. G.L. Carr, R.P.S.M. Lobo, J. LaVeigne, D.H. Reitze, D.B. Tanner, Phys. Rev. Lett. 85, 3001 (2000)

20. J. Demsar, R.D. Averitt, A.J. Taylor, V.V. Kabanov, W.N. Kang, H.J. Kim, E.M. Choi, S.I. Lee, Phys. Rev. Lett. 91, 267002 (2003)

21. R.P.S.M. Lobo, J.D. LaVeigne, D.H. Reitze, D.B. Tanner, Z.H. Barber, E. Jacques, P. Bosland, M.J. Burns, G.L. Carr, Phys. Rev. B 72, 024510 (2005)

22. M. Beck, M. Klammer, S. Lang, P. Leiderer, V.V. Kabanov, G.N. Gol'tsman, J. Demsar, Phys. Rev. Lett. 107, 177007 (2011)

23. C. Giannetti, M. Capone, D. Fausti, M. Fabrizio, F. Parmigiani, D. Mihailovic, Adv. Phys. 65, 58-238 (2016)

24. D.M. Ginsberg, Phys. Rev. Lett. 8, 204 (1962)

25. W.H. Parker, W.D. Williams, Phys. Rev. Lett. 29, 924 (1972)

26. J.R. Schrieffer, D.M. Ginsberg, Phys. Rev. Lett. 8, 207 (1962)

27. B.I. Miller, A.H. Dayem, Phys. Rev. Lett. 8, 207 (1967)

28. K.E. Gray, A.R. Long, C.J. Adkins, Philos. Mag. 20, 273 (1969)

29. A. Rothwarf, B.N. Taylor, Phys. Rev. Lett. 19, 27 (1967)

30. L.R. Testardi, Phys. Rev. B 4, 2189 (1971)

31. C.S. Owen, D.J. Scalapino, Phys. Rev. Lett. 28, 1559 (1972)

32. G.A. Sai-Halasz, C.C. Chi, A. Denenstein, D.N. Langenberg, Phys. Rev. Lett. 33, 215 (1974)

33. W. Parker, Phys. Rev. B 12, 3667 (1975)

34. V.F. Elesin, YuV Kopaev, Sov. Phys. Usp. 24, 116 (1981)

35. A. Schmid, Phys. Rev. Lett. 38, 922 (1977)

36. G.M. Eliashberg, Pis'ma ZETF 11, 186 (1970). [JETP Lett. 11, 114 (1970)]

37. B.I. Ivlev, S.G. Lisitsyn, G.M. Eliashberg, J. Low Temp. Phys. 10, 449 (1973)

38. I. Rousseau, MSc Thesis, University of Konstanz (2012)

39. U. Eckern, A. Schmid, M. Schmutz, G. Schon, J. Low Temp. Phys. 36, 643 (1979)

40. J.J. Chang, D.J. Scalapino, Phys. Rev. B 15, 2651 (1977)

41. J.J. Chang, D.J. Scalapino, J. Low Temp. Phys. 29, 477 (1977)

42. J.J. Chang, D.J. Scalapino, J. Low Temp. Phys. 31, 1 (1978)

43. T.J. Tredwell, E.H. Jacobsen, Phys. Rev. Lett. 35, 244 (1975)

44. T.J. Tredwell, E.H. Jacobsen, Phys. Rev. B 13, 2931 (1976)

45. D. Seligson, J. Clarke, Phys. Rev. B 28, 6297 (1983)

46. N.C. Cirillo Jr., W.L. Clinton, J.R. Waterman, Phys. Rev. B 25, 5698 (1982)

47. J.A. Pals, K. Weiss, P. van Attekum, R.E. Horstman, J. Wolter, Phys. Rep. 89, 323 (1979)

48. J.E. Mooij, Nonequilibrium Superconductivity, Phonons, and Kapitza Boundaries (Springer, Berlin, 1981), p. 191

49. D.J. Hilton, in Optical Techniques for Solid-State Materials Characterization, ed by R.P. Prasankumar, A.J. Taylor (Francis \& Taylor, New York, 2011)

50. J. Demsar, R. Hudej, J. Karpinski, V.V. Kabanov, D. Mihailovic, Phys. Rev. B 63, 054519 (2001)

51. N. Gedik, M. Langner, J. Orenstein, S. Ono, Y. Abe, Y. Ando, Phys. Rev. Lett. 95, 117005 (2005)

52. P. Kusar, V.V. Kabanov, J. Demsar, T. Mertelj, S. Sugai, D. Mihailovic, Phys. Rev. Lett. 101, 227001 (2008)

53. C. Giannetti, G. Zgrablic, C. Consani, A. Crepaldi, D. Nardi, G. Ferrini, G. Dhalenne, A. Revcolevschi, F. Parmigiani, Phys. Rev. B 80, 235129 (2009)

54. T. Mertelj, V.V. Kabanov, C. Gadermaier, N.D. Zhigadlo, S. Katrych, J. Karpinski, D. Mihailovic, Phys. Rev. Lett. 102, 17002 (2009)

55. E.E.M. Chia, D. Talbayev, J.-X. Zhu, H.Q. Yuan, T. Park, J.D. Thompson, C. Panagopoulos, G.F. Chen, J.L. Luo, N.L. Wang, A.J. Taylor, Phys. Rev. Lett. 104, 027003 (2010)

56. B. Mansart, D. Boschetto, A. Savoia, F. Rullier-Albenque, F. Bouquet, E. Papalazarou, A. Forget, D. Colson, A. Rousse, M. Marsi, Phys. Rev. B 82, 024513 (2010)

57. D.H. Torchinsky, G.F. Chen, J.L. Luo, N.L. Wang, N. Gedik, Phys. Rev. Lett. 105, 027005 (2010)

58. R.A. Kaindl, M.A. Carnahan, D.S. Chemla, S. Oh, J.N. Eckstein, Phys. Rev. B 72, 060510 (2005)

59. A. Pashkin, M. Porer, M. Beyer, K.W. Kim, A. Dubroka, C. Bernhard, X. Yao, Y. Dagan, R. Hackl, A. Erb, J. Demsar, R. Huber, A. Leitenstorfer, Phys. Rev. Lett. 105, 067001 (2010) 
60. M. Beyer, D. Städter, M. Beck, H. Schäfer, V.V. Kabanov, G. Logvenov, I. Bozovic, G. Koren, J. Demsar, Phys. Rev. B 83, 214515 (2011)

61. M. Beck, M. Klammer, I. Rousseau, M. Obergfell, P. Leiderer, M. Helm, V.V. Kabanov, I. Diamant, A. Rabinowicz, Y. Dagan, J. Demsar, Phys. Rev. B 95, 085106 (2017)

62. M. Šindler, C. Kadlec, P. Kužel, K. Ilin, M. Siegel, H. Nemec, Phys. Rev. B 97, 054507 (2018)

63. L. Perfetti, P.A. Loukakos, M. Lisowski, U. Bovensiepen, H. Eisaki, M. Wolf, Phys. Rev. Lett. 99, 197001 (2007)

64. R. Cortés, L. Rettig, Y. Yoshida, H. Eisaki, M. Wolf, U. Bovensiepen, Phys. Rev. Lett. 107, 097002 (2011)

65. C.L. Smallwood, J.P. Hinton, C. Jozwiak, W. Zhang, J.D. Koralek, H. Eisaki, D.-H. Lee, J. Orenstein, A. Lanzara, Science 336, 1137 (2012)

66. W. Lee, J.J. Lee, E.A. Nowadnick, W. Tabis, S.W. Huang, V.N. Strocov, E.M. Motoyama, G. Yu, B. Moritz, M. Greven, T. Schmitt, Z.X. Shen, T.P. Devereaux, Nat. Phys. 10, 883-889 (2014)

67. Yu. He, I.M. Vishik, M. Yi, S. Yang, Z. Liu, J.J. Lee, S. Chen, S.N. Rebec, D. Leuenberger, A. Zong, C.M. Jefferson, R.G. Moore, P.S. Kirchmann, A.J. Merriam, Z.-X. Shen, Rev. Sci. Inst. 87, 011301 (2016)

68. S. Parham, H. Li, T.J. Nummy, J.A. Waugh, X.Q. Zhou, J. Griffith, J. Schneeloch, R.D. Zhong, G.D. Gu, D.S. Dessau, Phys. Rev. X 7, 041013 (2017)

69. I. Avigo et al., Phys. Status Solidi B 254, 1600382 (2017)

70. M. Beck, H. Schäfer, G. Klatt, J. Demsar, S. Winnerl, M. Helm, T. Dekorsy, Opt. Express 18, 9251 (2010)

71. W. Zimmermann, E.H. Brandt, M. Bauer, E. Seider, L. Genzel, Physica C 183, 99 (1991)

72. P.B. Allen, Phys. Rev. Lett. 59, 1460 (1987)

73. S.D. Brorson, A. Kazeroonian, J.S. Moodera, D.W. Face, T.K. Cheng, E.P. Ippen, M.S. Dresselhaus, G. Dresselhaus, Phys. Rev. Lett. 64, 2172 (1990)

74. J. Demsar, T. Dekorsy, in Optical Techniques for Solid-State Materials Characterization, ed by R.P. Prasankumar, A.J. Taylor (Francis \& Taylor, New York, 2011)

75. V.V. Baranov, V.V. Kabanov, Phys. Rev. B 89, 125102 (2014)

76. M. Obergfell, J. Demsar, Phys. Rev. Lett. 124, 037401 (2020)

77. A. Rothwarf, G.A. Sai-Halasz, D.N. Langensberg, Phys. Rev. Lett. 33, 212 (1974)

78. V.V. Kabanov, J. Demsar, D. Mihailovic, Phys. Rev. Lett. 95, 147002 (2005)

79. YuN Ovchinnikov, V.Z. Kresin, Phys. Rev. B 58, 12416 (1998)

80. C. Geibel, H. Rietschel, A. Junod, M. Pelizzone, J. Muller, J. Phys. F Met. Phys. 15, 405 (1985)

81. W. Weber, Phys. Rev. B 8, 5082 (1973)

82. U. Haufe, G. Kerker, K.H. Bennemann, Sol. Stat. Comm. 17, 321 (1975)

83. N. Gedik, P. Blake, R.C. Spitzer, J. Orenstein, R. Liang, D.A. Bonn, W. Hardy, Phys. Rev. B 70, 014504 (2004)

84. J.P. Hinton, E. Thewalt, Z. Alpichshev, F. Mahmood, J.D. Koralek, M.K. Chan, M.J. Veit, C.J. Dorow, N. Barišić, A.F. Kemper, D.A. Bonn, W.N. Hardy, Ruixing Liang, N. Gedik, M. Greven, A. Lanzara, J. Orenstein, Sci. Rep. 6, 23610 (2016)

85. J. Orenstein, Phys. Today 65, 44 (2012)

86. S. Sobolev, T. Dong, N. Bhattacharjee, A. Lanz, A.R. Pokharel, et al., in preparation

87. K.S. Il'in, M. Lindgren, M. Currie, A.D. Semenov, G.N. Gol'tsman, Roman Sobolewski, Appl. Phys. Lett. 76, 2752 (2000)

88. J. Demsar, V.K. Thorsmolle, J.L. Sarrao, A.J. Taylor, Phys. Rev. Lett. 96, 037401 (2006)

89. J. Demsar, J.L. Sarrao, A.J. Taylor, J. Phys. Condens. Matter 18, R281 (2006)

90. K.H. Ahn, M.J. Graf, S.A. Trugman, J. Demsar, R.D. Averitt, J.L. Sarrao, A.J. Taylor, Phys. Rev. B 69, 045114 (2004)

91. J. Demsar, R.D. Averitt, K.H. Ahn, M.J. Graf, S.A. Trugman, V.V. Kabanov, J.L. Sarrao, A.J. Taylor, Phys. Rev. Lett. 91, 027401 (2003)

92. K.S. Burch, E.E.M. Chia, D. Talbayev, B.C. Sales, D. Mandrus, A.J. Taylor, R.D. Averitt, Phys. Rev. Lett. 100, 026409 (2008)

93. J. Demsar, V.V. Kabanov, A.S. Alexandrov, H.J. Lee, E.D. Bauer, J.L. Sarrao, A.J. Taylor, Phys. Rev. B 80, 085121 (2009)

94. E.E.M. Chia, Jian-Xin Zhu, D. Talbayev, H.J. Lee, Namjung Hur, N.O. Moreno, R.D. Averitt, J.L. Sarrao, A.J. Taylor, Phys. Rev. B 84, 174412 (2011)

95. D. Mihailovic, B. Podobnik, J. Demsar, G. Wagner, J. Evetts, J. Phys. Chem. Solids 59, 1937 (1998) 
96. I. Schuller, K.E. Gray, Phys. Rev. Lett. 36, 429 (1976)

97. A. Schmid, G. Schoen, J. Low Temp. Phys. 20, 207 (1975)

98. D. Dvorsek et al., Phys. Rev. B 66, 020510 (2002)

99. P. Kusar, J. Demsar, D. Mihailovic, S. Sugai, Phys. Rev. B 72, 014544 (2005)

100. Y.H. Liu, Y. Toda, K. Shimatake, N. Momono, M. Oda, M. Ido, Phys. Rev. Lett. 101, 137003 (2008)

101. C.W. Luo, H.P. Lo, C.H. Su, I.H. Wu, Y.-J. Chen, K.H. Wu, J.-Y. Lin, T.M. Uen, J.Y. Juang, T. Kobayashi, Phys. Rev. B 82, 104512 (2010)

102. Y. Toda, F. Kawanokami, T. Kurosawa, M. Oda, I. Madan, T. Mertelj, V.V. Kabanov, D. Mihailovic, Phys. Rev. B 90, 094513 (2014)

103. S. Kumar, L. Harnagea, S. Wurmehl, B. Buechner, A.K. Sood, EPL 105, 47004 (2014)

104. T. Naito, Y. Yamada, T. Inabe, Y. Toda, J. Phys. Soc. Jpn. 77, 064709 (2008)

105. D. Mihailovic, V.V. Kabanov, K. Žagar, J. Demsar, Phys. Rev. B 60, R6995(R) (1999)

106. M.L. Schneider et al., Europhys. Lett. 60, 460 (2002)

107. E.J. Nicol, J.P. Carbotte, Phys. Rev. B 67, 214506 (2003)

108. P.C. Howell, A. Rosch, P.J. Hirschfeld, Phys. Rev. Lett. 92, 037003 (2004)

109. T. Matsuzaki, N. Momono, M. Oda, M. Ido, J. Phys. Soc. Jpn. 73, 2232 (2004)

110. M. Beck, I. Rousseau, M. Klammer, P. Leiderer, M. Mittendorff, S. Winnerl, M. Helm, G.N. Gol'tsman, J. Demsar, Phys. Rev. Lett. 110, 267003 (2013)

111. D. Fausti, R.I. Tobey, N. Dean, S. Kaiser, A. Dienst, M.C. Hoffmann, S. Pyon, T. Takayama, H. Takagi, A. Cavalleri, Science 331, 189 (2011)

112. W. Hu, S. Kaiser, D. Nicoletti, C.R. Hunt, I. Gierz, M.C. Hoffmann, M. Le Tacon, T. Loew, B. Keimer, A. Cavalleri, Nat. Mater. 13, 705 (2014)

113. R. Mankowsky, A. Subedi, M. Först, S.O. Mariager, M. Chollet, H.T. Lemke, J.S. Robinson, J.M. Glownia, M.P. Minitti, A. Frano, M. Fechner, N.A. Spaldin, T. Loew, B. Keimer, A. Georges, A. Cavalleri, Nature 516, 71 (2014)

114. S. Kaiser, C.R. Hunt, D. Nicoletti, W. Hu, I. Gierz, H.Y. Liu, M. Le Tacon, T. Loew, D. Haug, B. Keimer, A. Cavalleri, Phys. Rev. B 89, 184516 (2014)

115. M. Mitrano, A. Cantaluppi, D. Nicoletti, S. Kaiser, A. Perucchi, S. Lupi, P. Di Pietro, D. Pontiroli, M. Ricco, S.R. Clark, D. Jaksch, A. Cavalleri, Nature 530, 461 (2016)

116. J. Demsar, Nat. Phys. 12, 202 (2016)

117. A. Cantaluppi, M. Buzzi, G. Jotzu, D. Nicoletti, M. Mitrano, D. Pontiroli, M. Riccò, A. Perucchi, P. Di Pietro, A. Cavalleri, Nat. Phys. 14, 837 (2018)

118. M. Budden, T. Gebert, M. Buzzi, G. Jotzu, E. Wang, T. Matsuyama, G. Meier, Y. Laplace, D. Pontiroli, M. Riccò, F. Schlawin, D. Jaksch, A. Cavalleri, arXiv:2002.12835

119. M. Buzzi, D. Nicoletti, M. Fechner, N. Tancogne-Dejean, M. A. Sentef, A. Georges, M. Dressel, A. Henderson, T. Siegrist, J. A. Schlueter, K. Miyagawa, K. Kanoda, M.-S. Nam, A. Ardavan, J. Coulthard, J. Tindall, F. Schlawin, D. Jaksch, A. Cavalleri, arXiv:2001.05389

120. M. Först, C. Manzoni, S. Kaiser, Y. Tomioka, Y. Tokura, R. Merlin, A. Cavalleri, Nat. Phys. 7, 854 (2011)

121. A. Cavalleri, Contemp. Phys. 59, 31-46 (2018)

122. K.A. Cremin, J. Zhang, C.C. Homes, G.D. Guc, Z. Suna, M.M. Foglera, A.J. Millis, D.N. Basov, R.D. Averitt, Proc. Natl. Acad. Sci. 116, 19875-19879 (2019)

123. J. Orenstein, J.S. Dodge, Phys. Rev. B 92, 134507 (2015)

124. D. Nicoletti, M. Mitrano, A. Cantaluppi, A. Cavalleri, arXiv: 1506.07846

125. H. Niwa, N. Yoshikawa, K. Tomari, R. Matsunaga, D. Song, H. Eisaki, R. Shimano, Phys. Rev. B 100, 104507 (2019)

126. S.J. Zhang, Z.X. Wang, H. Xiang, X. Yao, Q.M. Liu, L.Y. Shi, T. Lin, T. Dong, D. Wu, N.L. Wang, Phys. Rev. X 10, 011056 (2020)

127. M. Babadi, M. Knap, I. Martin, G. Refael, E. Demler, Phys. Rev. B 96, 014512 (2017)

128. N. Bittner, T. Tohyama, S. Kaiser, D. Manske, J. Phys. Soc. Jpn. 88, 044704 (2019)

129. A. Nava, C. Giannetti, A. Georges, E. Tosatti, M. Fabrizio, Nat. Phys. 14, 154 (2018)

130. G. Chiriacò, A.J. Millis, I.L. Aleiner, Phys. Rev. B 98, 220510(R) (2018)

131. G. Chiriacò, A.J. Millis, I.L. Aleiner, Phys. Rev. B 101, 041105(R) (2020)

132. S.J. Zhang, Z.X. Wang, L.Y. Shi, T. Lin, M.Y. Zhang, G.D. Gu, T. Dong, N.L. Wang, Phys. Rev. B 98, 020506(R) (2018)

133. S.J. Zhang, Z.X. Wang, D. Wu, Q.M. Liu, L.Y. Shi, T. Lin, S.L. Li, P.C. Dai, T. Dong, N.L. Wang, Phys. Rev. B 98, 224507 (2018) 
134. B. Liu, M. Först, M. Fechner, D. Nicoletti, J. Porras, T. Loew, B. Keimer, A. Cavalleri, Phys. Rev. X 10, 011053 (2020)

135. D.M. Kennes, E.Y. Wilner, D.R. Reichman, A.J. Millis, Nat. Phys. 13, 479 (2017)

136. M. Kim, Y. Nomura, M. Ferrero, P. Seth, O. Parcollet, A. Georges, Phys. Rev. B 94, 155152 (2016)

137. G. Mazza, A. Georges, Phys. Rev. B 96, 064515 (2017)

138. K. Allen, F. Hellman, Phys. Rev. B 60, 11765 (1999)

139. Z. Sun, M.M. Fogler, D.N. Basov, A.J. Millis, arXiv:2001.03704v1

140. D. Pekker, C.M. Varma, Annu. Rev. Condens. Matter Phys. 6, 269 (2015)

141. R. Sooryakumar, M.V. Klein, Phys. Rev. Lett. 45, 660 (1980)

142. P.B. Littlewood, C.M. Varma, Phys. Rev. Lett. 47, 811 (1981)

143. M.-A. Méasson, Y. Gallais, M. Cazayous, B. Clair, P. Rodière, L. Cario, A. Sacuto, Phys. Rev. B 89, 060503(R) (2014)

144. A.F. Volkov, Sh.M. Kogan, Zh. Eksp. Teor. Fiz. 65, 2038-2046 (1973) [Sov. Phys.-JETP 38, 1018 (1974)]

145. E.A. Yuzbashyan, O. Tsyplyatyev, B.L. Altshuler, Phys. Rev. Lett. 96, 097005 (2006)

146. T. Papenkort, V.M. Axt, T. Kuhn, Phys. Rev. B 76, 224522 (2007)

147. R. Matsunaga, Y.I. Hamada, K. Makise, Y. Uzawa, H. Terai, Z. Wang, R. Shimano, Phys. Rev. Lett. 111, 057002 (2013)

148. R. Shimano, N. Tsuji, Annu. Rev. Condens. Matter Phys. 11, 103-24 (2020)

149. R. Matsunaga, N. Tsuji, H. Fujita, A. Sugioka, K. Makise, Y. Uzawa, H. Terai, Z. Wang, H. Aoki, R. Shimano, Science 345, 1145 (2014)

150. T. Cea, C. Castellani, L. Benfatto, Phys. Rev. B 93, 180507(R) (2016)

151. T. Cea, P. Barone, C. Castellani, L. Benfatto, Phys. Rev. B 97, 094516 (2018)

152. Y. Murotani, R. Shimano, Phys. Rev. B 99, 224510 (2019)

153. M. Silaev, Phys. Rev. B 99, 224511 (2019)

154. A. Moor, A.F. Volkov, K.B. Efetov, Phys. Rev. Lett. 118, 047001 (2017)

155. S. Nakamura, Y. Iida, Y. Murotani, R. Matsunaga, H. Terai, R. Shimano, Phys. Rev. Lett. 122, 257001 (2019)

156. H. Krull, N. Bittner, G.S. Uhrig, D. Manske, A.P. Schnyder, Nat. Commun. 7, 11921 (2016)

157. M.A. Müller, P.A. Volkov, I. Paul, I.M. Eremin, Phys. Rev. B 100, 140501(R) (2019)

158. F. Giorgianni, T. Cea, C. Vicario, C.P. Hauri, W.K. Withanage, X. Xi, L. Benfatto, Nat. Phys. 15, 341 (2019)

159. A.K. Mills, S. Zhdanovich, M.X. Na, F. Boschini, E. Razzoli, M. Michiardi, A. Sheyerman, M. Schneider, T.J. Hammond, V. Sü ss, C. Felser, A. Damascelli, D.J. Jones, Rev. Sci. Inst. 90, 083001 (2019)

160. D. Kutnyakhov et al., Rev. Sci. Inst. 91, 013109 (2020)

161. C. Lee, T. Rohwer, E.J. Sie, A. Zong, E. Baldini, J. Straquadine, P. Walmsley, D. Gardner, Y.S. Lee, I.R. Fisher, N. Gedik, Rev. Sci. Instrum. 91, 043102 (2020)

Publisher's Note Springer Nature remains neutral with regard to jurisdictional claims in published maps and institutional affiliations. 